TRANSACTIONS OF THE

AMERICAN MATHEMATICAL SOCIETY

Volume 364, Number 2, February 2012, Pages 961-1005

S 0002-9947(2011)05488-7

Article electronically published on September 15, 2011

\title{
DYNAMICS OF STRONGLY COMPETING SYSTEMS WITH MANY SPECIES
}

\author{
E. N. DANCER, KELEI WANG, AND ZHITAO ZHANG
}

\begin{abstract}
In this paper, we prove that the solution of the Lotka-Volterra competing species system with strong competition converges to a stationary point under some natural conditions. We also study the moving boundary problem of the singular limit equation, which plays an important role in our proof.
\end{abstract}

\section{Contents}

1. Introduction

2. The singular limit: Stationary case

3. The singular limit: Parabolic case

4. Asymptotics in strong competition

5. Approximate Clean Up Lemma

5.1. Systems with zeroth order terms

5.2. Proof of the Approximate Clean Up Lemma

5.3. A linearized version 997

5.4. A boundary version 1002

Acknowledgement $\quad 1004$

References

\section{INTRODUCTION}

The Lotka-Volterra model of competing species describes the competition of a number of species in a fixed domain. Its general form is as follows:

$$
\begin{cases}\frac{\partial u_{i}}{\partial t}-\Delta u_{i}=f_{i}\left(u_{i}\right)-u_{i} \sum_{j \neq i} b_{i j} u_{j}, & \text { in } \Omega \times(0,+\infty), \\ u_{i}=0\left(\text { or } \frac{\partial u_{i}}{\partial n}=0\right), & \text { on } \partial \Omega \times(0,+\infty), \\ u_{i}=\phi_{i}, & \text { on } \Omega \times\{0\},\end{cases}
$$

Received by the editors April 8, 2010 and, in revised form, September 3, 2010. 2010 Mathematics Subject Classification. Primary 35B40, 35R35, 35K57, 92D25.

Key words and phrases. Competing species, reaction-diffusion system, free boundary problem.

This work was supported by the Australian Research Council and the National Natural Science Foundation of China (10831005, 10971046). 
where $b_{i j}>0$ are constants and $1 \leq i, j \leq M$, with $M$ the number of the species, and $\Omega$ is a bounded domain in $\mathbb{R}^{n}(n \geq 1)$ with smooth boundary. The intra-species constraint term $f_{i}\left(u_{i}\right)$ is usually the logistic model:

$$
f_{i}(t)=a_{i} t-t^{2}, \forall t \in \mathbb{R} .
$$

Usually we consider a homogeneous Dirichlet $u_{i}=0$ or Neumann boundary condition $\frac{\partial u_{i}}{\partial n}=0$, and we impose a consistent condition on the initial values $\phi_{i} \geq 0$. We only study nonnegative solutions, that is, $u_{i} \geq 0$ for all $i$. The study of this reaction-diffusion system has a long history and there exist a great many works on the subject. However, most of these works are concerned with the case of two species. As far as we know, study in the case of many competing species has been very limited. In the 1990s Dancer and Du studied three species of competition systems and received very interesting existence results. It is believed that generally this system has complicated dynamics (see [14], 15]), even in the ordinary differential equation cases (see [27]).

In recent years, people have shown much interest in strongly competing systems with many species, that is, the system (or its elliptic case)

$$
\frac{\partial u_{i}}{\partial t}-\triangle u_{i}=a_{i} u_{i}-u_{i}^{2}-\kappa u_{i} \sum_{j \neq i} b_{i j} u_{j}
$$

where $\kappa$ is sufficiently large. Conti, Terracini and Verzini [9], 10, Caffarelli, Karakhanyan and Lin [2] 3], etc., established the regularity of the singular limit (and the partial regularity of its free boundary) as $\kappa \rightarrow+\infty$ and the uniform regularity for all $\kappa>0$. Conti, Terracini and Verzini found that in the singular limit of the stationary problem, species are spatially segregated and satisfy a remarkable system of differential inequalities, and these two conditions are also satisfied by the solution of a variational problem. Although it's not fully established, it's very possible that this singular limit has a variational structure. That is, the solution of a corresponding elliptic problem (as the equations do not contain the terms $f_{i}\left(u_{i}\right)$ ) is the harmonic map from the domain $\Omega$ into a metric space $\Sigma$ with nonpositive curvature, which has been studied by many authors since the work of [20]. Here the metric space $\Sigma$ is defined as follows:

$$
\Sigma:=\left\{\left(u_{1}, u_{2}, \cdots, u_{M}\right) \in \mathbb{R}^{M}: u_{i} \geq 0, u_{i} u_{j}=0 \text { for } i \neq j\right\} .
$$

This harmonic map is the critical point (in the weak sense as in [9]) of the functional

$$
\sum_{i=1}^{M} \int_{\Omega}\left|\nabla u_{i}\right|^{2}
$$

defined in the class of functions $u=\left(u_{1}, u_{2}, \cdots, u_{M}\right) \in\left(H^{1}(\Omega)\right)^{M}$ satisfying $u_{i} \geq 0$ and $u_{i} u_{j}=0$, a.e.; see $[9]$.

In our original problem and the corresponding elliptic problem, a term $F(u)$ needs to be added to the above formulation:

$$
\sum_{i=1}^{M}\left(\frac{1}{2} \int_{\Omega}\left|\nabla u_{i}\right|^{2}-\int_{\Omega} F_{i}\left(u_{i}\right)\right),
$$

where $F_{i}(u)=\int_{0}^{u} f_{i}(s) d s$. The solution of the parabolic system of our singular limit could also be seen as a weak solution of the heat flow for the harmonic map (ignoring the zeroth order terms as above). 
So although our original problem in case $\kappa$ finite has no variational structure, its singular limit indeed has. Thus it's natural to conjecture (this is probably due to E. N. Dancer) that for $\kappa$ large, this system has simple dynamics, in particular, has no periodic solution. This is also related to an open problem of Weiming Ni concerning the existence of a periodic solution for fixed $\kappa$ (the report given at the Pacific Institute for the Mathematical Science, University of British Columbia, 2001.7).

This problem has been studied by Dancer and Zhang 18 for the case of two species. They proved that, under some natural assumptions on nondegeneracy, for $\kappa$ large and two species, in any dimension, any solution of the initial value problem will converge to a stationary solution as time $t$ tends to infinity.

By using some ideas from the recent work of Caffarelli, Karakhanyan and Lin [2, we obtain new results concerning this problem, with the number of species greater than 2 , in dimension 1 . Under some natural assumptions on nondegeneracy similar to those in [18, for $\kappa$ large, any solution of the initial value problem will converge to a stationary solution as time $t$ tends to infinity.

The problem we study in this paper is the following initial value problem with a homogeneous Dirichlet (or Neumann) boundary value condition in 1 dimension:

$$
\begin{cases}\frac{\partial u_{i}}{\partial t}-\frac{\partial^{2} u_{i}}{\partial x^{2}}=a_{i} u_{i}-u_{i}^{2}-\kappa u_{i} \sum_{j \neq i} b_{i j} u_{j} & \text { in }[0,1] \times(0,+\infty), \\ u_{i}(0)=u_{i}(1)=0\left(\text { or }\left.\frac{\partial u_{i}}{\partial x}\right|_{x=0,1}=0\right), & \\ u_{i} \geq 0 & \text { in }[0,1] \times(0,+\infty), \\ u_{i}=\phi_{i} & \text { on }[0,1] \times\{0\} .\end{cases}
$$

Here $i=1,2 \cdots, M, \phi_{i}$ are given Lipschitz continuous functions on [0,1] which satisfy

$$
\phi_{i} \geq 0, \text { and } \phi_{i} \phi_{j}=0 \text { for } i \neq j \text {. }
$$

In the paper, we will simply take $b_{i j} \equiv 1$ for simplicity. If there is any need to modify the argument arising from $b_{i j} \neq 1$, we will indicate it in the remark.

From the classical theory of reaction-diffusion systems (see, for example, 23]), we know that the solution $u_{i, \kappa}$ of (1.1) exists globally and is unique. Moreover, from the regularity results of [2] (see Theorem 1 and the Remark in the paper; this can also be proven using the blow up method of [10]), $\forall \alpha \in(0,1), u_{i, \kappa}$ are uniformly bounded for $\kappa$ in $C^{\alpha}([0,1] \times(0,+\infty))$. Here the distance function is the parabolic distance in $[0,1] \times(0,+\infty)$, defined as

$$
\operatorname{dist}\left(\left(t_{1}, x_{1}\right),\left(t_{2}, x_{2}\right)\right):=\left|t_{1}-t_{2}\right|^{\frac{1}{2}}+\left|x_{1}-x_{2}\right| \text {. }
$$

So we can say (up to a subsequence) that they converge uniformly in any compact subset of $[0,1] \times(0,+\infty)$ to $u_{i}$. More precisely, given any sequence $u_{i, \kappa_{n}}, \kappa_{n} \rightarrow+\infty$, we can extract a subsequence which converges to $u_{i}$ in the above sense; $u_{i}$ is also Hölder continuous. Similar to the elliptic case treated by S. Terracini et al. in [9] 
and [10, it is easily seen that $u_{i}$ satisfy $(1 \leq i, j \leq M)$

$(1.2)$

$$
\left\{\begin{array}{l}
\frac{\partial u_{i}}{\partial t}-\frac{\partial^{2} u_{i}}{\partial x^{2}} \leq a_{i} u_{i}-u_{i}^{2} \text { in }(0,1) \times(0,+\infty) \\
\left(\frac{\partial}{\partial t}-\frac{\partial^{2}}{\partial x^{2}}\right)\left(u_{i}-\sum_{j \neq i} u_{j}\right) \geq a_{i} u_{i}-u_{i}^{2}-\sum_{j \neq i}\left(a_{j} u_{j}-u_{j}^{2}\right) \text { in }(0,1) \times(0,+\infty) \\
u_{i}(0)=u_{i}(1)=0\left(\text { or }\left.\frac{\partial u_{i}}{\partial x}\right|_{x=0,1}=0\right) \\
u_{i}=\phi_{i} \text { on }[0,1] \times\{0\} \\
u_{i} \geq 0 ; u_{i} u_{j}=0 \text { for } i \neq j \text { in }[0,1] \times(0,+\infty)
\end{array}\right.
$$

The above differential inequalities are always understood in the weak sense (and in this paper, this kind of differential inequalities are always understood in the weak sense), that is, e.g., $\forall \varphi(x, t) \geq 0$ smooth with compact support in $(0,1) \times(0,+\infty)$, for the first inequality we have $(\forall i, 1 \leq i \leq M)$

$$
\int_{0}^{+\infty} \int_{0}^{1}-\frac{\partial \varphi}{\partial t} u_{i}+\frac{\partial u_{i}}{\partial x} \frac{\partial \varphi}{\partial x}-\left(a_{i} u_{i}-u_{i}^{2}\right) \varphi d x d t \leq 0 .
$$

In 2, they also prove that the limit $u_{i}$ are locally Lipschitz continuous in the parabolic distance in $[0,1] \times(0,+\infty)$ (see their Theorem 22). In fact, $u_{i}$ are Lipschitz continuous in the parabolic distance since they are uniformly bounded in our case. Here we need to note that, although our equations look a little different from those in [2], their method can still be applied to our case, since the term $f_{i}\left(u_{i}\right)$ we add can be seen as a small perturbation after a rescaling. For the details, please see Section 4.1 below.

First we study the dynamics of (1.2): we show that after a finite period of time the set where $u_{i}$ is positive has only finitely many components and that the number of these is bounded. This is true even if at time zero the set where $u_{i}$ is positive has infinitely many components. After a period of time no extinction can happen any more. Finally, the $u_{i}$ converge to a stationary state as time $t$ goes to infinity, that is, if a species is sufficiently small compared with the other species, it will become extinct in the future after a fixed finite time.

Imposing some assumptions, for system (1.1) with strong competition (large $\kappa$ ), we can prove that for any initial value, the solution will eventually converge to a stationary state (see Theorem 4.11). The proof follows the idea of [18, but in our case we need some distinctly nontrivial technical changes.

Note that, different from the case with only two species, which can be transferred into a single equation by subtracting the two equations, if the number of species $M \geq 3$, there may exist interactions between all the species, and we can't transfer our equations into a single equation. We find that, in 1 dimension, due to the topological restriction, in most areas, locally it appears that there are only two species competing, that is, other species are very small compared to these two dominating species. This is essentially the Approximate Clean Up Lemma, which originates from the Clean Up Lemma in [2] and is valid in any dimension.

We also need a linearization version and a boundary version of the Approximate Clean Up Lemma. The proof of these various forms of the Clean Up Lemma follows from the powerful iteration scheme of [2]. In the paper [18, for the solutions of the linearized equation of (1.1), the authors used the Kato inequality to derive a 
uniform bound from the $L^{2}$ bound. This causes no restriction in 2 species, but if the number of species is more, unless we assume the symmetry $b_{i j}=b_{j i}$, the Kato inequality can't be applied directly. Here we can avoid this by our linearization version of the Approximate Clean Up Lemma. Possibly in one dimension we may find other more direct methods to attack this problem, but we still think that our treatment may be useful in higher dimensions.

At last we want to say something about our assumptions. In order to prove the convergence to a stationary point, we impose some nondegeneracy conditions (see Section 5). Some of these conditions are verified in one dimension. However, these conditions seem to be too restrictive in a higher dimension. Anyway, if we add these conditions in a higher dimension, most of the arguments in this paper can go through if we bypass the regularity of the free boundaries near the fixed boundary.

It seems that even if we do not impose these conditions artificially, we can still prove a weak version of the conjecture above (see Corollary 4.3 for the proof in the case of one dimension). More precisely, we show that for $\kappa$ large any periodic solution must stay near some stationary solution of the singular limit system. That is, it looks like a stationary solution which slowly changes in time. We will pursue this problem in the future.

The paper is organized as follows. In Section 2 we give a preliminary analysis of the stationary case of (1.2), establishing that there are only finitely many solutions. In Section 3 we study the system (1.2) and show that the solution converges to the stationary state (see Theorem 3.1). In the process of the proof we also describe the extinction phenomena. In Section 4 we establish an Approximate Clean Up Lemma and its linearization version and boundary version. In Section 5 we prove that, under some assumptions, the solution of (1.1) converges to a stationary state.

\section{The Singular limit: Stationary CASe}

In this section we study the stationary case of differential inequalities (1.2):

$$
\left\{\begin{array}{l}
-\frac{d^{2} u_{i}}{d x^{2}} \leq a_{i} u_{i}-u_{i}^{2} \text { in }(0,1) ; \\
-\frac{d^{2}}{d x^{2}}\left(u_{i}-\sum_{j \neq i} u_{j}\right) \geq a_{i} u_{i}-u_{i}^{2}-\sum_{j \neq i}\left(a_{j} u_{j}-u_{j}^{2}\right) \text { in }(0,1) ; \\
u_{i} \geq 0, u_{i}(0)=u_{i}(1)=0 ; \\
u_{i} u_{j}=0 \text { in }[0,1], \text { for } i \neq j(1 \leq i, j \leq M) .
\end{array}\right.
$$

The main result of this section is Theorem 2.4. The Neumann boundary value problem can be treated similarly. We know that the above problem can have a solution with some identically zero components, thus it may have multiple solutions, but if the set where $u_{i}$ is positive has several components, we treat these as different species (because we are in the stationary case). Here the number of species may become infinite. Below we exclude this case. We have the following theorem. (Note that Lemma 2.2 below ensures that the set where $u_{i}$ is positive has only finitely many components.)

Theorem 2.1. $\forall M \in \mathbb{N}$, there exists at most one solution $\left(u_{1}, u_{2}, \cdots, u_{M}\right)$ of (2.1) (up to permutation) such that each $u_{i}$ is not identically zero and each of its supports is an interval. 
First we need a well-known lemma. For later use and completeness we give the proof here.

Lemma 2.2. For each $L>\frac{\pi}{2 \sqrt{a}}$, there exists a unique positive solution $u$ of

$$
\left\{\begin{array}{l}
-\frac{d^{2} u}{d x^{2}}=a u-u^{2} \text { in }(0, L) \\
u(0)=u(L)=0
\end{array}\right.
$$

There is no positive solution if $L \leq \frac{\pi}{2 \sqrt{a}}$.

Proof. The existence can be easily proven by, for example, the method of sub- and sup-solutions. In fact, we have the following conservation quantity:

$$
\left(\frac{d u}{d x}\right)^{2}+a u^{2}-\frac{2}{3} u^{3} \equiv c,
$$

for some constant $c$.

If we have two positive solutions $u$ and $v$, then on the open set $D:=\{u>v\}$ (if not empty) we have

$$
\begin{aligned}
0 & \geq \int_{\partial D} \frac{\partial u}{\partial \nu} v-\frac{\partial v}{\partial \nu} u \\
& =\int_{D} \Delta u v-\triangle v u \\
& =\int_{D}-\left(a u-u^{2}\right) v+\left(a v-v^{2}\right) u \\
& =\int_{D} u v(u-v) \\
& >0
\end{aligned}
$$

which is a contradiction.

Corollary 2.3. The constant $c$ defined in (2.3), seen as a function of $L$, is increasing in $L$.

Proof. Assume $L_{1}>L_{2}>\frac{\pi}{2 \sqrt{a}}$ with $u_{1}$ and $u_{2}$ the solution of (2.2) in $\left[0, L_{1}\right]$ and $\left[0, L_{2}\right]$, respectively. Because

$$
0=u_{2}\left(L_{2}\right)<u_{1}\left(L_{2}\right)
$$

the same method as in the previous lemma gives that in $\left[0, L_{2}\right]$,

$$
u_{1}>u_{2} \text {. }
$$

This implies

$$
\frac{d u_{1}}{d x}(0)>\frac{d u_{2}}{d x}(0) .
$$

Note that here we have the strict inequality because otherwise we will have $u_{1} \equiv u_{2}$. Because

$$
c_{i}\left(L_{i}\right)=\left(\frac{d u_{i}}{d x}(0)\right)^{2}
$$

our claim follows. 
Now we give the proof of Theorem 2.1

Proof. Since each support $\left\{u_{i}>0\right\}$ is an interval, and for $i \neq j,\left\{u_{i}>0\right\}$ and $\left\{u_{j}>0\right\}$ are disjoint (since $u_{i} u_{j}=0$ ), we can assume

$$
\left\{u_{i}>0\right\}=\left(\alpha_{i}, \beta_{i}\right),
$$

with (by renumbering if necessary)

$$
0 \leq \alpha_{1}<\beta_{1} \leq \alpha_{2}<\beta_{2} \leq \cdots \leq \alpha_{m}<\beta_{m} \leq 1 .
$$

Because on the set $\left(\beta_{i-1}, \alpha_{i+1}\right)$, where all $u_{j} \equiv 0$ for $j \neq i, u_{i}$ satisfies the equation (by combining the first and second equation of (2.1))

$$
-\frac{d^{2} u_{i}}{d x^{2}}=a_{i} u_{i}-u_{i}^{2}
$$

Moreover, $u_{i} \geq 0$ and $u_{i}$ does not vanish identically on this interval, so we must have $u_{i}>0$ in $\left(\beta_{i-1}, \alpha_{i+1}\right)$. Thus

$$
\alpha_{i+1}=\beta_{i} \text { and } \alpha_{1}=0, \beta_{m}=1,
$$

that is, the set where all $u_{i}$ vanish contains at most finitely many points.

In $\left(\alpha_{i}, \beta_{i}\right)$, there exists a constant $c_{i}>0$ such that

$$
\left(\frac{d u_{i}}{d x}\right)^{2}+a_{i} u_{i}^{2}-\frac{2}{3} u_{i}^{3}=c_{i} .
$$

Because $u_{i}\left(\alpha_{i}\right)=u_{i}\left(\beta_{i}\right)=0$, then

$$
\left(\frac{d u_{i}}{d x}\left(\alpha_{i}\right)\right)^{2}=c_{i}=\left(\frac{d u_{i}}{d x}\left(\beta_{i}\right)\right)^{2} .
$$

In $\left(\alpha_{i}, \beta_{i+1}\right)$, from the second inequalities in (2.1)

$$
\left\{\begin{array}{l}
-\frac{d^{2}}{d x^{2}}\left(u_{i}-u_{i+1}\right) \geq a_{i} u_{i}-u_{i}^{2}-\left(a_{i+1} u_{i+1}-u_{i+1}^{2}\right) \text { in }\left(\alpha_{i}, \beta_{i+1}\right), \\
-\frac{d^{2}}{d x^{2}}\left(u_{i+1}-u_{i}\right) \geq a_{i+1} u_{i+1}-u_{i+1}^{2}-\left(a_{i} u_{i}-u_{i}^{2}\right) \text { in }\left(\alpha_{i}, \beta_{i+1}\right),
\end{array}\right.
$$

we see that

$$
-\frac{d^{2}\left(u_{i}-u_{i+1}\right)}{d x^{2}}=a_{i} u_{i}-u_{i}^{2}-a_{i+1} u_{i+1}+u_{i+1}^{2} .
$$

This implies $u_{i}-u_{i+1} \in C^{1}\left(\alpha_{i}, \beta_{i+1}\right)$. Then we have

$$
\left(\frac{d u_{i}}{d x}\left(\beta_{i}\right)\right)^{2}=\left(\frac{d u_{i+1}}{d x}\left(\beta_{i}\right)\right)^{2} .
$$

So all of the $c_{i}$ in (2.5) are the same, which we denote by $c$. Now from Corollary 2.3 we know that each $\beta_{i}-\alpha_{i}$ is uniquely determined by $c$ (because it is a strictly increasing function of $c$ ). Then there exists at most one $c$ such that

$$
\sum_{i}\left(\beta_{i}-\alpha_{i}\right)=1 \text {. }
$$

Theorem 2.4. There are only finitely many solutions of (2.1). 
Proof. It's well known that if $u>0$ in a bounded domain $\Omega$ with boundary value 0 and if

$$
-\triangle u=a u-u^{2}
$$

then

$$
a>\lambda_{1}
$$

where $\lambda_{1}$ is the first eigenvalue of $\Omega$ with a Dirichlet boundary condition.

Now in our case each connected component of $u_{i}$, which is an interval, has length $\geq \frac{\pi}{2 \sqrt{a_{i}}}$. So the total number of connected components of each possible solution of (2.1) has an upper bound, which depends on those $a_{i}, 1 \leq i \leq M$. By Theorem 2.1 we can finish the proof.

\section{The Singular limit: Parabolic CASE}

In this section, we study the dynamics of the singular limit equation (1.2). The main result is the following Theorem 3.1. With a similar idea, we also give a description of the connecting orbit (Proposition 3.12) and a uniqueness result of the initial value problem (Proposition 3.14).

Theorem 3.1. Let's define

$$
m(T):=\#\left\{\text { connected components of } \bigcup_{i}\left\{u_{i}>0\right\} \cap\{t=T\}\right\} .
$$

Then we have:

(1) $m(T)$ is a nonincreasing function of $T$.

(2) There exist at most countable points $T_{1}>T_{2}>\cdots$ with $T_{k} \rightarrow 0$, which are all of the places where the value of $m(T)$ jumps.

(3) For $t \in\left(T_{i+1}, T_{i}\right)$ (here we define $\left.T_{0}=+\infty\right)$, we can rearrange the indices $i$ (where we can throw away those species vanishing identically here) such that if we define

$$
u:=u_{1}-u_{2}+u_{3}-u_{4}+\cdots,
$$

it satisfies the following equation:

$$
\left(\frac{\partial}{\partial t}-\frac{\partial^{2}}{\partial x^{2}}\right) u=f,
$$

where $f:=a_{1} u_{1}-u_{1}^{2}-\left(a_{2} u_{2}-u_{2}^{2}\right)+\cdots$ is a Lipschitz continuous function.

(4) In $[0,1] \times\left(T_{1},+\infty\right)$ we have $\lim _{t \rightarrow+\infty} u_{i}(x, t)=v_{i}(x)$, where $v:=\left(v_{1}, v_{2}, \cdots\right)$ is a solution of (2.1). Moreover, either $v \equiv 0$ or $v$ is nontrivial and changes sign exactly $m\left(T_{1}\right)$ times.

First, we show a kind of regularizing property of (1.2). This is a special case of the Dimension Reduction Principle (see, for example, [6]). However, it is not written explicitly in the literature, so we will give the arguments here for completeness.

Proposition 3.2. For each $t>0$, for the free boundary $\mathcal{F}(u):=\bigcup_{i} \partial\left\{u_{i}>0\right\}$ in the problem of (1.2), $\mathcal{F}(u) \cap\{t\}$ contains only finitely many points.

Proof. Assume for some $t>0$ that there exist infinitely many points in $\mathcal{F}(u) \cap$ $\{t\}$. Take a $x_{0} \in \mathcal{F}(u) \cap\{t\} \cap(0,1)$ such that there exist $x_{k} \neq x_{0}$ in $\mathcal{F}(u)$ and 
$\lim _{k \rightarrow \infty} x_{k}=x_{0}$. Without loss of generality, we can assume $x_{0}=0$ and $t=0$. (We can change the domain by translation.)

Take

$$
r_{k}=x_{k}-x_{0}
$$

which we can assume to be a positive number. Now define the blow up sequence

$$
u^{k}(x, t):=\frac{1}{L_{k}} u\left(r_{k} x, r_{k}^{2} t\right),
$$

where $L_{k}=\frac{1}{\left|C_{r_{k}}\right|} \int_{C_{r_{k}}}|u|^{2}$ and $C_{r_{k}}=\left[-r_{k}, r_{k}\right] \times\left[-r_{k}^{2}, r_{k}^{2}\right]$ is the parabolic cylinder.

As in [2] (or Theorem 2.1 in [6]), we know there exists a subsequence (still denoted by $\left.u^{k}\right)$ converging to $v(x, t)$ locally uniformly on $\mathbb{R}^{1} \times(-\infty,+\infty)$, with $v$ nontrivial and nonnegative. Moreover, there exists a positive integer $d$, which is the frequency of $(0,0)$ (for the definition and its property, please see Section 9 in [2]), such that in $\mathbb{R}^{1} \times(-\infty,+\infty)$,

$$
\left\{\begin{array}{l}
\frac{\partial v_{i}}{\partial t}-\frac{\partial^{2} v_{i}}{\partial x^{2}} \leq 0 \\
\left(\frac{\partial}{\partial t}-\frac{\partial^{2}}{\partial x^{2}}\right)\left(v_{i}-\sum_{j \neq i} v_{j}\right) \geq 0 \\
v_{i} v_{j}=0 \text { for } i \neq j \\
v_{i}\left(\lambda x, \lambda^{2} t\right)=\lambda^{d} v_{i}(x, t) .
\end{array}\right.
$$

Because $(1,0) \in \mathcal{F}\left(u^{k}\right)$, we also have $v_{i}(1,0)=0$ for all $i$, or equivalently $(1,0) \in$ $\mathcal{F}(v)$. Then from the homogeneity of $v$ we have

$$
\mathbb{R}_{+}^{1} \times\{0\} \subset \mathcal{F}(v) .
$$

This contradicts the dimension estimate of the free boundary. (From Section 10 of [2] and Theorem 2.3 in [6], we know that the Hausdorff dimension of the free boundary restricted to time $t=0$ is 0 .)

If $x_{0}$ lies on the fixed boundary, the blow up limit $v$ is defined on $\mathbb{R}_{+}^{1} \times(-\infty,+\infty)$, with the boundary condition

$$
v=0, \text { on }\{0\} \times(-\infty,+\infty) .
$$

This can be treated similarly.

Now we have the following lemma:

Lemma 3.3. $\forall T_{1}>T_{2}>0$, any connected component of $\left\{u_{i}>0\right\} \cap\left\{t<T_{1}\right\}$ must intersect with $\left\{t=T_{2}\right\}$.

Proof. We know that $\left\{u_{i}>0\right\}$ is an open subset of $[0,1] \times[0,+\infty)$, so it is locally path connected and the path connected components coincide with the connected components. Assume there exists a connected component $A$, with $\left\{t=T_{2}\right\} \cap A=\emptyset$.

Now consider the restriction $\hat{u}_{i}$ of $u_{i}$ on $A$. First, it is still continuous. So we have

$$
\begin{cases}\frac{\partial \hat{u}_{i}}{\partial t}-\frac{\partial^{2} \hat{u}_{i}}{\partial x^{2}} \leq a_{i} \hat{u}_{i}-\hat{u}_{i}^{2} & \text { in }\left\{\hat{u}_{i}>0\right\} \\ \hat{u}_{i} \geq 0 & \text { in }[0,1] \times\left[T_{2}, T_{1}\right], \\ \hat{u}_{i}=0 & \text { on } \partial_{p}\left([0,1] \times\left[T_{2}, T_{1}\right]\right),\end{cases}
$$


where $\partial_{p}\left([0,1] \times\left[T_{2}, T_{1}\right]\right)$ is the parabolic boundary. Now we must have $\hat{u}_{i} \equiv 0$. In fact, take a positive constant $C>a_{i}$ and define

$$
v(x, t):=e^{-C t} \hat{u}_{i}(x, t) .
$$

We have in the open set $\{v>0\}$

$$
\begin{aligned}
\frac{\partial v}{\partial t}-\frac{\partial^{2} v}{\partial x^{2}} & \leq e^{-C t}\left(-C \hat{u}_{i}+a_{i} \hat{u}_{i}-\hat{u}_{i}^{2}\right) \\
& <0 .
\end{aligned}
$$

If $\hat{u}_{i}$ is not identically zero, then $\max _{[0,1] \times\left[T_{2}, T_{1}\right]} v$ is attained at some point $\left(x_{0}, t_{0}\right)$ where $\hat{u}_{i}\left(x_{0}, t_{0}\right)>0$. We know $\hat{u}_{i}$ is positive in a neighborhood of $\left(x_{0}, t_{0}\right)$ because $\hat{u}_{i}$ is continuous. Then $v$ is smooth in this neighborhood, and we can apply the maximum principle to get a contradiction.

Remark 3.4. From the proof, we see that this lemma remains true in higher dimensions and with a more general nonlinearity.

Without loss of generality, we can assume that as $t=T^{\prime}>0, u_{i}$ satisfy the condition that $\left\{u_{i}>0\right\} \cap\left\{t=T^{\prime}\right\}$ is an interval for each $i$. Later it will be clear that, as time evolves, two connected components may merge into one but a single connected component can’t split into two.

Corollary 3.5. $\forall T>T^{\prime},\left\{u_{i}>0\right\} \cap\{t=T\}$ is an interval if it is not empty.

Proof. If $\exists T>0$, such that $\left\{u_{i}>0\right\} \cap\{t=T\}$ contains two disjoint intervals $\left(\alpha_{1}, \beta_{1}\right) \cup\left(\alpha_{2}, \beta_{2}\right)$ where

$$
\alpha_{1}<\beta_{1}<\alpha_{2}<\beta_{2}
$$

and

$$
\left(\beta_{1}, \alpha_{2}\right) \cap\left\{u_{i}>0\right\}=\emptyset .
$$

From Lemma 3.3 we know there exist two continuous paths $\gamma_{1}(t)$ and $\gamma_{2}(t)$ which start from a point in $\left\{u_{i}>0\right\} \cap\left\{t=T^{\prime}\right\}$ and end in two points lying in $\left(\alpha_{1}, \beta_{1}\right) \times\{T\}$ and $\left(\alpha_{2}, \beta_{2}\right) \times\{T\}$, respectively.

There exists some $j \neq i$ such that

$$
\left(\left(\beta_{1}, \alpha_{2}\right) \times\{T\}\right) \cap\left\{u_{j}>0\right\} \neq \emptyset .
$$

However, this set is contained in a connected component which can't be connected to $\left\{t=T^{\prime}\right\}$ through a continuous path, contradicting Lemma 3.3, and we conclude the proof of the corollary.

Now the first and the second parts of Theorem 3.1 are easily seen. The points $T_{k}$ are exactly where some components of $u_{i}$ become extinct.

Assume in $\left(T_{i+1}, T_{i}\right)$ that $u_{1}, u_{2}, \cdots, u_{m}$ are not identically zero, where $m \leq M$. With the help of Lemma 3.3. we can show that any two connected components of $\left\{u_{i}>0\right\}$ and $\left\{u_{j}>0\right\}$ with $i \neq j$ can't interweave, that is, if at $t_{1} \in\left(T_{i+1}, T_{i}\right)$, $\left\{u_{i}>0\right\}$ lies above $\left\{u_{j}>0\right\}$, then at another $t_{2} \in\left(T_{i+1}, T_{i}\right),\left\{u_{i}>0\right\}$ can't lie below $\left\{u_{j}>0\right\}$. So we can rearrange the index properly so that $\left\{u_{i}>0\right\}$ is adjacent to $\left\{u_{i-1}>0\right\}$ and $\left\{u_{i+1}>0\right\}$ (here we treat the same species with 
different domains as different species; this causes no confusion in $\left.\left(T_{i+1}, T_{i}\right)\right)$. Now the third part of Theorem 3.1 is proved. Now in this time interval we have

Lemma 3.6. $\forall t \in\left(T_{i+1}, T_{i}\right)$, there exist

$$
0=\alpha_{0}(t) \leq \alpha_{1}(t) \leq \cdots<\alpha_{m}(t)=1
$$

such that

$$
\left\{u_{j}>0\right\} \cap\{t\}=\left(\alpha_{j-1}(t), \alpha_{j}(t)\right) .
$$

Moreover, all the $\alpha_{j}(t)$ are continuous functions of $t$.

Proof. From Corollary 3.5 we know that for each $t>0$ there exist

$$
0=\alpha_{0}(t)<\beta_{1}(t) \leq \alpha_{1}(t)<\beta_{2}(t) \leq \cdots<\beta_{m}(t)=1
$$

such that

$$
\left\{u_{j}>0\right\} \cap\{t\}=\left(\alpha_{j-1}(t), \beta_{j}(t)\right) .
$$

From the unique continuation property of the linear parabolic equation

$$
\frac{\partial u}{\partial t}-\frac{\partial^{2} u}{\partial x^{2}}=V u
$$

where

$$
V:=\left(a_{1}-u_{1}\right)-\left(a_{2}-u_{2}\right)+\cdots
$$

is an $L^{\infty}$ function, we know that, $\forall t$, the set $\{x: u(x, t)=0\}$ can't contain an open set. From this, we easily see

$$
\beta_{j}(t)=\alpha_{j}(t) .
$$

Now $\bigcup_{i}\left\{\left(\alpha_{j}(t), t\right): t \in\left(T_{i+1}, T_{i}\right)\right\}$ is the nodal set of $u$, and the continuity of $\alpha_{j}(t)$ is easily seen by its local uniqueness.

Remark 3.7. In the proof we need the fact that $u$ is not identically zero in $[0,1] \times$ $\left(T_{i+1}, T_{i}\right)$. This can be guaranteed by the unique continuation property of the linear parabolic equation (3.4) (see 26]; note here we have zero boundary condition). Note that although the form of the equation changes when crossing those extinction time $T_{i}$, if $u_{i} \equiv 0$ in $\left\{t>T_{i}\right\}$, then $u_{i} \equiv 0$ at $t=T_{i}$ and we can apply the backward uniqueness in $\left(T_{i+1}, T_{i}\right]$ again.

These curves are in fact regular. That is,

Proposition 3.8. $\forall t \in\left(T_{i+1}, T_{i}\right)$, at $\left(\alpha_{j}(t), t\right)$, we have (here $u$ is defined as in Theorem 3.1)

$$
\frac{\partial u}{\partial x} \neq 0 .
$$

In particular, $\alpha_{j}(t)$ are $C^{1}$ in $t$.

Proof. Note that since $u$ satisfies (3.4) and that both $V$ and $u$ are bounded, standard parabolic estimates imply that $u$ is $C^{1}$ in the space variable $x$. So the formulation in the proposition makes sense.

We need the characterization of the singular points of the free boundaries (see Section 9 of [2] or [6]). Assuming we are at $(0,0)$, we have a positive integer number $d$ (the frequency) such that the blow up sequences

$$
u_{\lambda}(x, t)=\lambda^{-d} u\left(\lambda^{2} t, \lambda x\right)
$$


converge to $v(x, t)$ as $\lambda \rightarrow 0$, which is a polynomial of the form (the Hermitian polynomial)

$$
\sum_{k=0}^{\left[\frac{d}{2}\right]} \frac{d !}{k !(d-2 k) !} x^{d-2 k} t^{k}
$$

Moreover, if $\frac{\partial u}{\partial x}=0$, then $d \geq 2$. In other words, $\frac{\partial u}{\partial x} \neq 0$ is equivalent to $d=1$.

Now, $\{v(x, t)=0\}$ is composed of $d$ nonsingular curves $C_{i}$ which have the form

$$
t=-c_{i} x^{2}, 1 \leq i \leq d
$$

Here $c_{i}$ are the zeros of the corresponding Hermite polynomials, and "nonsingular" means at these points $\frac{\partial v}{\partial x} \neq 0$ (this is because Hermite polynomials have simple zeros).

Now because $u_{\lambda}$ converges to $v$ in $C^{1}$, by the inverse function theorem we know for $\lambda$ small near each $C_{i}$ we have a nonsingular nodal curve of $u_{\lambda}$. In particular, there exist two points $x_{1, \lambda} \neq x_{2, \lambda}$ such that

$$
u_{\lambda}\left(-1, x_{1, \lambda}\right)=u_{\lambda}\left(-1, x_{2, \lambda}\right)=0,
$$

if $\lambda$ small enough. Coming back to $u$, this means

$$
u\left(-\lambda^{2}, \lambda x_{1, \lambda}\right)=u\left(-\lambda^{2}, \lambda x_{2, \lambda}\right)=0 .
$$

This is a contradiction, because from the previous lemma we have near $(0,0)$ that the nodal curve of $u_{\lambda}$ (that is, of $u$ ) is a single continuous curve, which implies there exists a unique $\alpha\left(-\lambda^{2}\right)$ such that

$$
u\left(-\lambda^{2}, \alpha\left(-\lambda^{2}\right)\right)=0 .
$$

Remark 3.9. Similar results to Proposition 3.8 already appeared in 6]. See also Section 10 in [2].

With this regularity property of the free boundary we can give an "energy identity". Define the energy

$$
E(t):=\sum_{i} \int_{[0,1]} \frac{1}{2}\left|\frac{\partial u_{i}}{\partial x}\right|^{2}-\frac{1}{2} a_{i} u_{i}^{2}+\frac{1}{3} u_{i}^{3} .
$$

We have

Proposition 3.10. For $t \in\left(T_{i+1}, T_{i}\right)$

$$
\frac{d}{d t} E(t)=-\sum_{i} \int_{[0,1]}\left|\frac{\partial u_{i}}{d t}\right|^{2} .
$$

In particular, $E(t)$ is decreasing in $t$. 
Proof. We have

$$
\begin{aligned}
& \frac{d}{d t} \int_{\alpha_{i}(t)}^{\beta_{i}(t)} \frac{1}{2}\left|\frac{\partial u_{i}}{\partial x}\right|^{2}-\frac{1}{2} a_{i} u_{i}^{2}+\frac{1}{3} u_{i}^{3} \\
= & \int_{\alpha_{i}(t)}^{\beta_{i}(t)}\left(\frac{\partial u_{i}}{\partial x} \frac{\partial^{2} u_{i}}{\partial x \partial t}-a_{i} u_{i} \frac{\partial u_{i}}{\partial t}+u_{i}^{2} \frac{\partial u_{i}}{\partial t}\right) d t \\
& -\left[\frac{1}{2}\left(\frac{\partial u_{i}}{\partial x}\right)^{2}-\frac{1}{2} u_{i}^{2}+\frac{1}{3} u_{i}^{3}\right]\left(\alpha_{i}(t), t\right) \frac{d \alpha_{i}(t)}{d t} \\
& +\left[\frac{1}{2}\left(\frac{\partial u_{i}}{\partial x}\right)^{2}-\frac{1}{2} u_{i}^{2}+\frac{1}{3} u_{i}^{3}\right]\left(\beta_{i}(t), t\right) \frac{d \beta_{i}(t)}{d t} \\
= & -\int_{\alpha_{i}(t)}^{\beta_{i}(t)} \frac{\partial u_{i}}{\partial t}\left[\frac{\partial^{2} u_{i}}{\partial x^{2}}+a_{i} u_{i}-u_{i}^{2}\right] \\
& +\frac{\partial u_{i}}{\partial x}\left(\beta_{i}(t), t\right) \frac{\partial u_{i}}{\partial t}\left(\beta_{i}(t), t\right)-\frac{\partial u_{i}}{\partial x}\left(\alpha_{i}(t), t\right) \frac{\partial u_{i}}{\partial t}\left(\alpha_{i}(t), t\right) \\
& -\frac{1}{2}\left|\frac{\partial u_{i}}{\partial x}\right|^{2}\left(\alpha_{i}(t), t\right) \frac{d \alpha_{i}(t)}{d t}+\frac{1}{2}\left|\frac{\partial u_{i}}{\partial x}\right|^{2}\left(\beta_{i}(t), t\right) \frac{d \beta_{i}(t)}{d t} .
\end{aligned}
$$

By differentiating $u\left(\alpha_{i}(t), t\right)=0$ and noting the definition of $u$ we have

$$
\frac{\partial u_{i}}{\partial t}\left(\alpha_{i}(t), t\right)+\frac{\partial u_{i}}{\partial x}\left(\alpha_{i}(t), t\right) \frac{d \alpha_{i}(t)}{d t}=0 .
$$

So we get

$$
\frac{\partial u_{i}}{\partial x} \frac{\partial u_{i}}{\partial t}\left(\beta_{i}(t), t\right)=-\left|\frac{\partial u_{i}}{\partial x}\right|^{2}\left(\beta_{i}(t), t\right) \frac{d \beta_{i}(t)}{d t}
$$

and

$$
\frac{\partial u_{i}}{\partial x} \frac{\partial u_{i}}{\partial t}\left(\alpha_{i}(t), t\right)=-\left|\frac{\partial u_{i}}{\partial x}\right|^{2}\left(\alpha_{i}(t), t\right) \frac{d \alpha_{i}(t)}{d t} .
$$

Note that we have $\alpha_{i}(t)=\beta_{i-1}(t)$. Summing (3.9) up in $i$, we get the result.

The fourth part of Theorem 3.1 can be proved using the same method in [18]. The main idea is that if the solution is very close to a stationary solution for some time, then it either stays near this stationary solution all the time or it leaves away and never comes back. Here we will establish a property which, roughly speaking, can be said to be "no extinction at infinity".

From the discussion above, we know for a solution of (1.2) there exists a $T_{1}>0$, such that for all $t>T_{1}$, no extinction occurs. But at this stage we can't exclude the possibility that some $u_{i} \rightarrow 0$ as $t \rightarrow+\infty$ while remaining positive in any finite time. Now let's consider this problem:

Proposition 3.11. For each $i, u_{i}(x, t)$ converges to $u_{i}(x)$. If $\left(u_{1}(x), u_{2}(x), \cdots\right)$ are not identically zero, then each $u_{i}(x)$ is not identically zero.

Proof. From the energy identity (3.8), we know that

$$
\lim _{t \rightarrow+\infty} \int_{t}^{+\infty} \int_{0}^{1}\left|\frac{\partial u}{\partial t}\right|^{2}=0 .
$$

Because

$$
\left(\frac{\partial}{\partial t}-\frac{\partial^{2}}{\partial x^{2}}\right) \frac{\partial u}{\partial t}=\hat{V} \frac{\partial u}{\partial t}
$$


where $\hat{V}:=\left(a_{1}-2 u_{1}\right)-\left(a_{2}-2 u_{2}\right)+\cdots$ is uniformly bounded, by the standard parabolic estimate we get

$$
\lim _{t \rightarrow+\infty} \sup _{x}\left|\frac{\partial u}{\partial t}(x, t)\right|=0
$$

So we have at time $t$

$$
\sup _{x}\left|\frac{\partial^{2} u_{i}}{\partial x^{2}}+a_{i} u_{i}-u_{i}^{2}\right| \leq \epsilon(t)
$$

where $\lim _{t \rightarrow+\infty} \epsilon(t)=0$. From the statement about the Lipschitz regularity of solutions of (1.2), $\left|\frac{\partial u_{i}}{\partial x}\right|$ are uniformly bounded. We can multiply (3.16) with it to get

$$
\sup _{x}\left|\frac{\partial^{2} u_{i}}{\partial x^{2}} \frac{\partial u_{i}}{\partial x}+a_{i} u_{i} \frac{\partial u_{i}}{\partial x}-u_{i}^{2} \frac{\partial u_{i}}{\partial x}\right| \leq C \epsilon(t),
$$

for some constant $C$ independent of $t$. Integrating this in $x$ we get, for $x \in$ $\left(\alpha_{i}(t), \beta_{i}(t)\right)$,

$$
\left|\left[\frac{1}{2}\left(\frac{\partial u_{i}}{\partial x}\right)^{2}+\frac{1}{2} a_{i} u_{i}^{2}-\frac{1}{3} u_{i}^{3}\right](x, t)-\left[\frac{1}{2}\left(\frac{\partial u_{i}}{\partial x}\right)^{2}+\frac{1}{2} a_{i} u_{i}^{2}-\frac{1}{3} u_{i}^{3}\right]\left(\alpha_{i}(t), t\right)\right| \leq C \epsilon(t),
$$

where $\alpha_{i}(t)$ can be replaced by $\alpha_{i+1}(t)$. From this, if we define

$$
C(t):=\left[\frac{1}{2}\left(\frac{\partial u_{i}}{\partial x}\right)^{2}+\frac{1}{2} a_{i} u_{i}^{2}-\frac{1}{3} u_{i}^{3}\right]\left(\alpha_{i}(t), t\right)
$$

for some fixed $i$, then there exists a constant $C>0$ such that for all $i$ (similar to the elliptic case considered in Section 2),

$$
\left|\left[\frac{1}{2}\left(\frac{\partial u_{i}}{\partial x}\right)^{2}+\frac{1}{2} a_{i} u_{i}^{2}-\frac{1}{3} u_{i}^{3}\right](x, t)-C(t)\right| \leq C \epsilon(t) .
$$

In particular, if $C(t)$ is uniformly bounded from below by a fixed positive constant, then at time $t$, if we are at the maximal point of $u_{i}(x, t)$, because

$$
\frac{\partial u_{i}}{\partial x}=0,
$$

we have

$$
\left|\left[\frac{1}{2} a_{i} u_{i}^{2}-\frac{1}{3} u_{i}^{3}\right](x, t)-C(t)\right| \leq C \epsilon(t) .
$$

We can solve this equation to obtain

$$
\left|\max _{x} u_{i}(x, t)-C_{i}(t)\right| \leq C \epsilon(t),
$$

where $C_{i}(t)$ are bounded from below because they only depend on $C(t)$ and $a_{i}$. So no $u_{i}(x, t)$ can converge to 0 for any $t \rightarrow+\infty$.

On the other hand, if $\liminf _{t \rightarrow+\infty} C(t)=0$, then we have for a sequence $t_{k} \rightarrow+\infty$ that $\lim _{k \rightarrow+\infty} u\left(x, t_{k}\right)=0$. Moreover, from the standard parabolic estimate this convergence can be taken in $C^{1}$ topology, and we have for this sequence

$$
\lim _{t_{k} \rightarrow+\infty} \sum_{i} \int_{\Omega} \frac{1}{2}\left|\frac{\partial u_{i}}{\partial x}\right|^{2}-\frac{1}{2} a_{i} u_{i}^{2}+\frac{1}{3} u_{i}^{3}=0 .
$$


From the energy decreasing property (3.8), we then have

$$
\lim _{t \rightarrow+\infty} \sum_{i} \int_{\Omega} \frac{1}{2}\left|\frac{\partial u_{i}}{\partial x}\right|^{2}-\frac{1}{2} a_{i} u_{i}^{2}+\frac{1}{3} u_{i}^{3}=0 .
$$

In this case, we must have for all $i$ that

$$
\lim _{t \rightarrow+\infty} u_{i}(x, t)=0 .
$$

This is because any nontrivial solution of the stationary equation (2.1) has energy $<0$ strictly, which can be seen by an integration by parts.

The above method can be used to describe the entire solution, which is defined for all time as $t \in(-\infty,+\infty)$. First, from the energy decreasing property we have either

$$
\lim _{t \rightarrow-\infty} E(t)=+\infty
$$

or

$$
\lim _{t \rightarrow-\infty} E(t)<+\infty
$$

If the latter happens, we in fact have the limit as $t \rightarrow-\infty$ exists, that is,

Proposition 3.12. For a solution $u$ of (1.2) defined in $[0,1] \times(-\infty,+\infty)$, if

$$
\lim _{t \rightarrow-\infty} E(t)<+\infty
$$

then there exists a stationary solution $v$ of (1.2) (that is, a solution of (2.1)) such that

$$
\lim _{t \rightarrow-\infty} u_{i}(t)=v_{i}
$$

Proof. From the energy identity we have

$$
\int_{-\infty}^{0} \int_{[0,1]} \sum_{i}\left|\frac{\partial u_{i}}{d t}\right|^{2}<+\infty .
$$

In particular,

$$
\lim _{t \rightarrow-\infty} \int_{-\infty}^{t} \int_{[0,1]} \sum_{i}\left|\frac{\partial u_{i}}{d t}\right|^{2}=0 .
$$

Because $u_{i}(t)$ are uniformly Lipschitz continuous functions on $[0,1]$ (note here we have a control of the energy; see [2]), for any sequence $t_{k} \rightarrow-\infty$ there exists a subsequence converging to a Lipschitz continuous function $v_{i}$ for all $i$.

We claim that $\left(v_{i}\right)$ is a solution of (2.1). In fact, define

$$
u_{i, k}(x, t)=u_{i}\left(x, t+t_{k}\right) .
$$

It's a solution of (1.2) defined on $[0,1] \times(-\infty,+\infty)$ and uniformly Lipschitz continuous, so it converges locally uniformly to a solution $v$ of (1.2). Also we have $u_{i, k}(x, 0) \rightarrow v_{i}(x)$, so

Moreover, we have

$$
v_{i}(x, 0)=v_{i}(x)
$$

$$
E\left(u_{k}, t\right)=E\left(u, t+t_{k}\right)
$$

So for $t>0$,

$$
E\left(u_{k},-t\right) \leq E\left(u_{k}, t\right)+\varepsilon(k, t),
$$


where $\lim _{k \rightarrow+\infty} \varepsilon(k, t)=0$ for any fixed $t$. By the uniform Lipschitz continuity, $\forall t$,

$$
\lim _{k \rightarrow+\infty} E\left(u_{k}, t\right)=E(v, t) .
$$

Passing to the limit in the inequality (3.23) and noting the energy decreasing property (3.8) (this can be applied to the current case), we get

$$
E(v,-t)=E(v, t) .
$$

Using the energy identity (3.8), we have

$$
\sum_{i} \int_{-t}^{t} \int_{[0,1]}\left|\frac{\partial v_{i}}{\partial t}\right|^{2}=0
$$

This means

$$
\frac{\partial v_{i}}{\partial t} \equiv 0
$$

That is, $v_{i}$ is the stationary solution of (1.2).

We know that the stationary solution of (1.2) is a finite set (Theorem 2.4), but from standard theory we know that the $\omega$-limit set of $u(x, t)$ as $t \rightarrow-\infty$ is a connected set, so it must be a single point. In other words, if there exist two limit points, then we can prove that the limit set contains a path connecting these two points, contradicting our previous claim. The complete argument is as follows: Take small neighborhoods $V_{1}$ and $V_{2}$ of the two limiting points $v_{1}, v_{2}$, and neighborhoods $V_{i}$ of all other stationary solutions $v_{i}$, which are disjoint. We know there exist $t_{k, 1}$ and $t_{k, 2}$ which converge to $-\infty$ such that

$$
u\left(t_{k, 1}\right) \in V_{1} \text { and } u\left(t_{k, 2}\right) \in V_{2} .
$$

From connectedness of the orbit $u(t)$ we know there exists $t_{k}$ lying between $t_{k, 1}$ and $t_{k, 2}$ such that

$$
u\left(t_{k}\right) \text { is not in } \bigcup_{i} V_{i} .
$$

But from the uniform Lipschitz continuity, $u\left(t_{k}\right)$ converge to a limit in $C[0,1]$, which is not a stationary point. This is a contradiction.

Remark 3.13. For any solution $w$ of (1.2) which exists on $[0,1] \times(-\infty,+\infty)$, assume that there exist $u$ and $v$, which are solutions of (2.1) such that

$$
\lim _{t \rightarrow-\infty} w_{i}(x, t)=u_{i}(x), \lim _{t \rightarrow+\infty} w_{i}(x, t)=v_{i}(x) .
$$

Then we have two cases:

(1) $u \equiv 0$ and $v$ is nontrivial.

(2) Both $u$ and $v$ are nontrivial, with the energy of $u$ strictly larger than that of $v$.

Now we consider the uniqueness of the initial value problem (1.2) in a special case:

Proposition 3.14. For the system (1.2), if the total number of connected components of the initial value $\phi$ is finite, then there exists a unique solution. 
Proof. The existence is obvious, that is, the solution can be constructed as the limit of the solutions of system (1.1) $(\kappa \rightarrow+\infty)$. We just need to prove the local uniqueness.

Assume there exist two local solutions of (1.2), $u_{i}$ and $v_{i}$. By Theorem 3.1, we know there exists some $\epsilon>0$, such that in $[0, \epsilon)$, the sum in $i$ of the number of connected components of the support of the component $u_{i}$ (or $v_{i}$ ) equals that of $\phi=$ $\left(\phi_{1}, \phi_{2}, \cdots, \phi_{M}\right)$, and the free boundaries are nonsingular curves (see Proposition 3.8).

Let

$$
d(u, v)=\sum_{i}\left|u_{i}-v_{i}\right|
$$

Using the Kato inequality

$$
\nabla|h|=\operatorname{sgn}(h) \nabla h, \text { a.e., } \triangle|h| \geq \operatorname{sgn}(h) \triangle h, \text { a.e. }
$$

we have

$$
\begin{aligned}
& \left(\frac{\partial}{\partial t}-\frac{\partial^{2}}{\partial x^{2}}\right) d(u, v) \\
\leq & \sum_{i} \operatorname{sgn}\left(u_{i}-v_{i}\right)\left(\frac{\partial}{\partial t}-\frac{\partial^{2}}{\partial^{2} x}\right)\left(u_{i}-v_{i}\right) \\
\leq & \sum_{i} \operatorname{sgn}\left(u_{i}-v_{i}\right)\left[a_{i}-\left(u_{i}+v_{i}\right)\right]\left(u_{i}-v_{i}\right) \\
\leq & C d(u, v) .
\end{aligned}
$$

Here $\operatorname{sgn}\left(u_{i}-v_{i}\right)$ is the signature of $u_{i}-v_{i}$ and $C$ is a constant depending only on $a_{i}$ and $\phi$. The second inequality is valid by the following argument: by the regularity of the free boundaries, in fact we have

$$
\left(\frac{\partial}{\partial t}-\frac{\partial^{2}}{\partial x^{2}}\right) u_{i}=a_{i} u_{i}-u_{i}^{2}+\sum_{\alpha_{i}(t)} \frac{\partial u_{i}}{\partial \nu} \delta_{\alpha_{i}(t)}
$$

where $\alpha_{i}(t)$ is the boundary of $\left\{u_{i}>0\right\}$ at time $t$ (it consists of finitely many points) and $\nu$ is the outward unit normal vector to $\partial\left\{u_{i}>0\right\}$ (although in 1 dimension this is trivial, we keep this notation for clearness), and $\delta$ is the Dirac measure supported on these points. Summing these terms in $i$ we find that in the interior these $\delta$ measures cancel each other out and we are left with the regular part, while those at boundary points 0 (or 1 ) have the form

$$
\operatorname{sgn}\left(u_{i}-v_{i}\right) \frac{\partial\left(u_{i}-v_{i}\right)}{\partial \nu} \delta_{0} .
$$

If near $x=0, u_{i}-v_{i}>0$, then $\frac{\partial\left(u_{i}-v_{i}\right)}{\partial \nu}(0) \leq 0$ and vice versa. So this is a nonpositive term, and in the inequality we can throw it away.

Now $d(u, v) \equiv 0$ at $t=0$. Then the maximum principle gives

$$
d(u, v) \equiv 0
$$

for all $t \in[0, \epsilon)$, that is,

$$
u_{i} \equiv v_{i}
$$




\section{Asymptotics in Strong COMpetition}

In this section, we study the asymptotic behavior of (1.1) for $\kappa$ large. From the analysis in Section 3, we know that the singular limit as $\kappa \rightarrow+\infty$ is a gradient system (having a variational structure), with its solution converging to the stationary state as $t \rightarrow+\infty$. So the natural question arises: does (1.1) for $\kappa$ large also behave like a gradient system? In this section we will show that with a few assumptions, the answer is yes: for $\kappa$ large the dynamics of (1.1) is simple.

For simplicity, we assume the coefficients $b_{i j}=1, \forall i \neq j$. Without this assumption, our proof is still valid with minor changes, due to the special property of dimension 1. In the following, we will point this out whenever necessary.

In the proof of the main result of this section, especially in Theorem 4.4. we need some technical results. These results give the uniform a priori estimate and the convergence of the solutions of the linearized equations of (1.1) as $\kappa \rightarrow+\infty$. The precise statement and proof of these results are found in the next section.

We need to impose some assumptions on the stationary equation of (2.1), exactly as in [18. From Section 2, we know any solution of (2.1) can be arranged properly so that if we define

$$
u:=u_{1}-u_{2}+\cdots,
$$

then $u$ satisfies

$$
-\frac{d^{2} u}{d x^{2}}=f(x, u) \text { on }[0,1], u(0)=u(1)=0
$$

where

$$
f(x, u)=a_{i} u-(-1)^{i+1} u^{2}, \text { for } x \in\left\{x \in[0,1] \mid u_{i}(x)>0\right\} .
$$

If $\exists b_{i j} \neq 1$, in the above definition of $u, u$ has the form $u_{1}-c_{1} u_{2}+c_{2} u_{2}-\cdots$, where $c_{1}, c_{2}, \cdots$ are constants depending only on $b_{i j}$. The following statement and proof can be changed accordingly.

Any solution $u$ of (4.1) is nondegenerate, that is, any solution $v$ of

$$
-v^{\prime \prime}=g(x, u) v \text { a.e. on }[0,1], v(0)=v(1)=0
$$

with boundary value 0 , is identically zero. Here

$$
g(x, u)=f^{\prime}(x, u)=a_{i}-2(-1)^{i+1} u, \text { for } x \in\left\{x \in[0,1] \mid u_{i}(x)>0\right\} .
$$

In fact, we know that $f(x, u)$ is differentiable in $u$ except at $u=0$, and $f_{u}^{\prime}(x, u)<$ $\frac{f(x, u)}{u}, u \neq 0, f(x, 0)=0$. (Note that nontrivial solutions of (4.1) only vanish on a set of measure zero so that the linearization (4.2) makes sense.)

If $z$ is a solution of (4.1) with $k$ interior zeroes, then $z^{\prime}$ is a solution of the linearized equation (4.2) and it must have at least $k+1$ zeroes in $(0,1)$, one between each two zeroes of $z$ in $[0,1]$.

Suppose that $v$ satisfies (4.2). By the Sturm comparison theorem (comparing with $\left.z^{\prime}\right) v$ has at least $k$ zeroes in $(0,1)$ (one between any two zeroes of $z^{\prime}$ ).

Now

$$
-z^{\prime \prime}-\frac{f(x, z)}{z} z=0 \text { a.e. on }[0,1], z(0)=z(1)=0
$$

and $z$ has $k$ interior zeroes. Hence 0 must be the $(k+1)$ th eigenvalue of the eigenvalue problem

$$
-h^{\prime \prime}-\frac{f(x, z)}{z} h=\lambda h \text { a.e. on }[0,1], h(0)=h(1)=0 .
$$


Since $f_{u}^{\prime}(x, u)<\frac{f(x, u)}{u}$ as $u \neq 0$ a.e. on $(0,1)$, it follows by eigenvalue comparison that the $(k+1)$ th eigenvalue of

$$
-v^{\prime \prime}-g(x, u) v=\lambda v \text { a.e. on }[0,1], v(0)=v(1)=0
$$

is positive. Thus if 0 is an eigenvalue, it must be the $l$ th eigenvalue where $l<k+1$. Hence the corresponding eigenfunction has less than $l-1$ interior zeroes, and so less than $k$ interior zeroes. This contradicts the first part of the proof, and hence 0 is not an eigenvalue, i.e., (4.2) has no nontrivial solutions.

We assume:

(1) The system of differential inequalities

$$
\left\{\begin{array}{l}
-\frac{d^{2} u_{i}}{d x^{2}} \leq a_{i} u_{i} \text { in }[0,1], \\
-\frac{d^{2}}{d x^{2}}\left(u_{i}-\sum_{j \neq i} u_{j}\right) \geq a_{i} u_{i}-\sum_{j \neq i} a_{j} u_{j} \text { in }[0,1], \\
u_{i}(0)=u_{i}(1)=0, \\
u_{i} \geq 0, u_{i} u_{j}=0 \text { for } i \neq j, \text { in }[0,1],(1 \leq i, j \leq M)
\end{array}\right.
$$

has no nontrivial solutions.

(Assume $\left(u_{i}\right)$ is a nontrivial solution (we still consider a function on disjoint supports as two distinct functions). Similar to Section 2, there exist $\alpha_{m}(1 \leq m \leq k$ for some positive integer $k)$ such that

$$
0=\alpha_{0}<\alpha_{1}<\alpha_{2}<\cdots<\alpha_{k}=1,
$$

and there exist constants $c_{m}>0, d_{m}=\frac{-\alpha_{m-1}}{\alpha_{m}-\alpha_{m-1}} \pi(1 \leq m \leq k)$ such that

$$
u_{i_{m}}(x)= \begin{cases}c_{m} \sin \left(\frac{\pi}{\alpha_{m}-\alpha_{m-1}} x+d_{m}\right), & \text { if } x \in\left(\alpha_{m-1}, \alpha_{m}\right), \\ 0, & \text { otherwise, }\end{cases}
$$

and if $j \neq i_{m}$ for all $m, u_{j} \equiv 0$. Moveover, $\forall m, c_{m+1} \sqrt{a_{i_{m+1}}}=c_{m} \sqrt{a_{i_{m}}}$ and $\alpha_{m}-\alpha_{m-1}=\frac{\pi}{\sqrt{a_{i_{m}}}}$. This last condition implies

$$
\sum_{m=1}^{k} \frac{\pi}{\sqrt{a_{i_{m}}}}=1
$$

This is very restrictive. So we can assume $\exists\left\{a_{i}, i=1,2, \cdots, M\right\}$ such that there is only trivial solution of (4.3).)

(2) Nonconstant (in time) bounded positive solutions of the following system $(i=1,2, \cdots, M)$,

$$
\left\{\begin{array}{l}
\frac{\partial u_{1}}{\partial t}-\frac{\partial^{2} u_{1}}{\partial x^{2}}=a_{1} u_{1}-u_{1} \sum_{j \neq 1} u_{j} \text { in }[0,1] \times(-\infty,+\infty), \\
\frac{\partial u_{2}}{\partial t}-\frac{\partial^{2} u_{2}}{\partial x^{2}}=a_{2} u_{2}-u_{2} \sum_{j \neq 2} u_{j} \text { in }[0,1] \times(-\infty,+\infty), \\
\cdots \ldots \\
\frac{\partial u_{M}}{\partial t}-\frac{\partial^{2} u_{M}}{\partial x^{2}}=a_{M} u_{M}-u_{M} \sum_{j \neq M} u_{j} \text { in }[0,1] \times(-\infty,+\infty), \\
u_{i}(0, t)=u_{i}(1, t)=0 \text { for } t \in(-\infty,+\infty),
\end{array}\right.
$$


approach distinct stationary solutions as $t \rightarrow \pm \infty$. The positive stationary solutions are hyperbolic, and there are no circuits of heteroclinic positive orbits.

(This condition has been studied in [18, where they construct an example (with two equations) satisfying this condition.)

(3) $a_{i}>\pi^{2}\left(\forall i=1,2, \cdots, M, \pi^{2}\right.$ is the first eigenvalue of $-w^{\prime \prime}=\lambda w, w(0)=$ $w(1)=0)$.

(Assumption 3 implies that the equation $(\forall i=1,2, \cdots, M)$

$$
\left\{\begin{array}{l}
-\frac{d^{2} u}{d x^{2}}=a_{i} u \text { in }(0,1), \\
u(0)=u(1)=0
\end{array}\right.
$$

has no positive solutions.)

Now we state our main result

Theorem 4.1. Under the above assumptions $(1)-(3)$, for $\kappa$ large, any solution of (1.1) converges to a stationary point as $t \rightarrow+\infty$.

First, we need to establish a theorem which is the analogue to Theorem 2 in [18]. However, their proof can't be directly applied here, so we need some improvements.

Theorem 4.2. $\forall \epsilon>0$, there exists $\kappa(\epsilon)$ such that if $\kappa>\kappa(\epsilon)$, then there is a stationary solution $w$ of (2.1) such that

$$
\left|u_{i}(x, t)-w_{i}(x)\right| \leq \epsilon
$$

for all $x \in[0,1]$ if $t$ is large.

Proof. Take the space $X:=C^{\alpha}\left([0,1], \mathbb{R}^{M}\right)$ for some fixed $\alpha \in(0,1)$. For the solution $u_{\kappa}$ define

$$
A_{\kappa}:=\bigcup_{t}\left\{u_{\kappa}(t), t \in[0,+\infty)\right\}
$$

Because $u_{\kappa}(t)$ are uniformly bounded in $C^{\beta}\left([0,1], \mathbb{R}^{M}\right)$ for any $\beta \in(0,1)$ with bounds independent of $\kappa$ and $t$ (see [2] for example; this can also be proved by the blow up method in [10]), we know that $\bigcup_{\kappa} A_{\kappa}$ is a pre-compact set of $X$. So we can define the limit set

$$
A:=\left\{u \in X: \exists v_{\kappa} \in A_{\kappa}, \text { such that } v_{\kappa} \rightarrow u \text { in } X\right\} .
$$

Claim 1. A is a compact, closed set composed of the connecting orbits of the limit equation (1.2) with corresponding stationary points.

Here we add a remark: because we are in the situation of an initial value problem, the "connecting orbit" may connect the initial value to a stationary point of (1.2). However, this only causes minor changes in the following proof, because the local compactness and uniform convergence still hold. The discussion below can be modified slightly to deal with this case; so we will omit it.

The compactness is from the fact that $\left\|v_{\kappa}\right\|_{C^{\beta}} \leq C(\beta)$ and $v_{\kappa} \rightarrow u$ in $C[0,1]$, which implies $\|u\|_{C^{\beta}} \leq C(\beta), \forall u \in A$.

The second claim that $A$ is invariant can be shown as follows. Aassume $u_{\kappa}=$ $u_{\kappa}\left(t_{\kappa}\right) \rightarrow u$. First we have for $i \neq j$

$$
\lim _{\kappa \rightarrow \infty} \sup _{[0,1] \times[0,+\infty)} u_{i, \kappa} u_{j, \kappa}=0,
$$


so $u_{i} u_{j} \equiv 0$ for $i \neq j$. Next define

$$
v_{\kappa}(t)=u_{\kappa}\left(t_{\kappa}+t\right)
$$

Then the standard method shows that $v_{\kappa}$ locally converges (in the sense of [5]) to a solution $v(t)$ of (1.2) with $v(0)=u$.

If $t_{\kappa}$ is bounded, then $v$ is defined on $\left[-T_{0},+\infty\right.$ ) for some $T_{0}>0$ (noting that for any $T>0, u_{\kappa}(t)$ converges uniformly on $\left.[0, T]\right)$. Here we must have $v\left(-T_{0}\right)=\varphi$, the initial value.

If $t_{\kappa} \rightarrow+\infty$, then $v$ is defined on $(-\infty,+\infty)$. We need to prove that $v$ is a connecting orbit. In view of Proposition 3.12 , we just need to prove that

$$
\lim _{t \rightarrow-\infty} E(v, t)<+\infty
$$

where the energy $E$ is defined before Proposition 3.10. This can be guaranteed by the uniform Lipschitz continuity of $u_{\kappa}(t)$ for all $\kappa>0$ and $t>0$, which implies $\exists C$, such that for any $t \in(-\infty,+\infty)$, the Lipschitz constant of $v(t)$ is bounded by $C$. However, if we are in the case $b_{i j} \neq b_{j i}$, we don't know whether the uniform Lipschitz continuity is true, so we need to proceed as follows.

In view of the monotonicity of $E(t)$ in the limit case, we just need to prove that

$$
\lim _{t \rightarrow-\infty} \int_{t}^{t+1} E(v, \tau) d \tau<+\infty .
$$

This can be seen by taking the limit in $\int_{t}^{t+1} E\left(u_{\kappa_{n}}, \tau\right) d \tau$. First we know that as a measure, $\kappa_{n} u_{i, \kappa_{n}} \sum_{j \neq i} u_{j, \kappa_{n}}$ converge weakly to a Radon measure $\nu_{i}$, which is supported on $\partial\left\{v_{i}>0\right\}$. With the convergence of $u_{\kappa_{n}}$ to $v$ in $C\left(\left[T_{1}, T_{2}\right] \times[0,1]\right)$ $\left(T_{1} \leq T_{2}\right.$ given), we get

$$
\begin{aligned}
& \lim _{n \rightarrow+\infty} \int_{T_{1}}^{T_{2}} \int_{[0,1]} \kappa_{n} u_{i, \kappa_{n}}^{2} \sum_{j \neq i} u_{j, \kappa_{n}} d x d t \\
& =\lim _{n \rightarrow+\infty} \int_{T_{1}}^{T_{2}} \int_{[0,1]} \kappa_{n} v_{i} u_{i, \kappa_{n}} \sum_{j \neq i} u_{j, \kappa_{n}} d x d t \\
& =\int_{T_{1}}^{T_{2}} \int_{[0,1]} v_{i} d \nu_{i} \\
& =0 .
\end{aligned}
$$

By multiplying the equation with $u_{i, \kappa_{n}}$, we get

$$
\begin{array}{r}
\int_{[0,1]} \frac{1}{2} u_{i, \kappa_{n}}^{2}\left(T_{2}\right)-\int_{[0,1]} \frac{1}{2} u_{i, \kappa_{n}}^{2}\left(T_{1}\right)+\int_{T_{1}}^{T_{2}} \int_{[0,1]}\left|\nabla u_{i, \kappa_{n}}\right|^{2} \\
=\int_{T_{1}}^{T_{2}} \int_{[0,1]} a_{i} u_{i, \kappa_{n}}^{2}-\frac{1}{3} u_{i, \kappa_{n}}^{3}-\kappa_{n} u_{i, \kappa_{n}}^{2} \sum_{j \neq i} u_{j, \kappa_{n}} .
\end{array}
$$

By taking the limit and noting that other terms converge, we get

$$
\lim _{n \rightarrow+\infty} \int_{T_{1}}^{T_{2}} \int_{[0,1]}\left|\nabla u_{i, \kappa_{n}}\right|^{2}=\int_{T_{1}}^{T_{2}} \int_{[0,1]}\left|\nabla v_{i}\right|^{2} .
$$


Moreover, from (4.6) we see that the above quantities are uniformly bounded depending on $T_{2}-T_{1}$ and $\sup \sum_{i} u_{i}$ only. Thus

$$
\int_{t}^{t+1} \int_{[0,1]} \sum_{i} \int_{[0,1]} \frac{1}{2}\left|\frac{\partial v_{i}}{\partial x}\right|^{2}-\frac{1}{2} a_{i} v_{i}^{2}+\frac{1}{3} v_{i}^{3}
$$

are uniformly bounded independent of $t$. This ends the verification of Claim 1 .

Now assume all the solutions of (2.1) are $\left\{w_{0}, w_{1}, \cdots\right\}$ (by Theorem 2.4 this is a finite set) with $w_{0}=0$ (that is, all of the components are identically zero). In the space $X$, take small open neighborhoods $V_{i}$ for each $w_{i}$, and take a neighborhood $U$ of $A$. We know that for $\kappa$ large, $A_{\kappa} \subset U$ (from compactness). We can also take an open neighborhood $V_{-1}$ of the initial value $\varphi$, and $\varphi$ is denoted by $w_{-1}$.

Claim 2. There exist two universal constants $T>T^{\prime}$ (depending on our choice of the open neighborhoods $V_{i}$ only) such that for any connecting orbit of (1.2), its time lying outside $\bigcup_{i} V_{i}$ is smaller than $T$ and greater than $T^{\prime}$.

This can be proved by a compactness argument (Note that all of the connecting orbits form a compact set and the stationary points are finite.)

Claim 3. $\forall \epsilon>0, \exists \kappa_{0}$, such that if $\kappa>\kappa_{0}$, then there exist some connecting orbits $v_{1}(t), v_{2}(t), \cdots$ of (1.2), with either $u_{\kappa}(t) \in \bigcup_{i} V_{i}$ or for some $t_{\kappa}$

$$
\sup _{\left[-\frac{T}{2}, \frac{T}{2}\right]}\left\|u_{\kappa}(t)-v_{i}\left(t-t_{\kappa}\right)\right\| \leq \epsilon .
$$

Here $T$ is the constant in Claim 2. This roughly says that outside $\bigcup_{i} V_{i}, u_{\kappa}(t)$ can't be rotating too much around the manifold composed of connecting orbits: it almost goes down with a connecting orbit directly. Later we will show that the number of these connecting orbits are finite, but at this stage we can't exclude that there is an infinite number of them. However, at least we can choose a countable number of these connecting orbits (each occupy a period of time $T$ ).

The proof of Claim 3 is easy: if $u_{\kappa}\left(t_{\kappa}\right) \rightarrow v \in A$ with $v$ not in $\bigcup_{i} V_{i}$, then from compactness, on $\left[-\frac{T}{2}, \frac{T}{2}\right]$

$$
u_{\kappa}\left(t+t_{\kappa}\right) \rightarrow v(t)
$$

where $v(0)=v$.

Claim 4. $\exists \delta>0$, depending only on our choice of $V_{i}$, such that for any connecting orbit $v$, if for every $t \in\left[-\frac{T^{\prime}}{2}, \frac{T^{\prime}}{2}\right], v(t)$ lies outside $\bigcup_{i} V_{i}$, then we have

$$
E\left(v, \frac{T^{\prime}}{2}\right) \leq E\left(v,-\frac{T^{\prime}}{2}\right)-\delta .
$$

This can be proven by compactness.

Claim 5. $\exists \kappa_{0}$, such that for $\forall \kappa \geq \kappa_{0}$, if for every $t \in\left[-\frac{T^{\prime}}{2}, \frac{T^{\prime}}{2}\right], u_{\kappa}(t)$ lies outside $\bigcup_{i} V_{i}$, then we have some $h>0$ independent of $\kappa$, such that

$$
\int_{\frac{T^{\prime}}{2}}^{\frac{T^{\prime}}{2}+h} E\left(u_{\kappa}, t\right) d t \leq \int_{-\frac{T^{\prime}}{2}}^{-\frac{T^{\prime}}{2}+h} E\left(u_{\kappa}, t\right) d t-\frac{\delta}{2} .
$$


Assume this is wrong. Then there exists a sequence $\kappa_{n} \rightarrow+\infty$ such that $u_{\kappa_{n}}$ is the solution of (1.1) with the fixed initial value $\phi$, and $u_{\kappa_{n}}\left(\left[t_{\kappa_{n}}-\frac{T^{\prime}}{2}, t_{\kappa_{n}}+\frac{T^{\prime}}{2}\right]\right)$ lie outside $\bigcup_{i} V_{i}$, but

$$
\int_{t_{\kappa_{n}}+\frac{T^{\prime}}{2}}^{t_{\kappa_{n}}+\frac{T^{\prime}}{2}+h} E\left(u_{\kappa}, t\right) d t \geq \int_{t_{\kappa_{n}}-\frac{T^{\prime}}{2}}^{t_{\kappa_{n}}-\frac{T^{\prime}}{2}+h} E\left(u_{\kappa}, t\right) d t-\frac{\delta}{2} .
$$

If we assume the uniform Lipschitz estimate, then for all $t \in\left[-\frac{T^{\prime}}{2}, \frac{T^{\prime}}{2}\right]$ and $n$, $u_{\kappa_{n}}\left(t_{\kappa_{n}}+t\right)$ are uniform Lipschitz continuous. So after taking a subsequence, it converges to a solution $v(t)$ of (1.2), which is defined on $\left[-\frac{T^{\prime}}{2}, \frac{T^{\prime}}{2}\right]$, and $v(t)$ lie outside $\bigcup_{i} V_{i}\left(\bigcup_{i} V_{i}\right.$ is an open set), and

$$
\int_{\frac{T^{\prime}}{2}}^{\frac{T^{\prime}}{2}+h} E(v, t) d t \geq \int_{-\frac{T^{\prime}}{2}}^{-\frac{T^{\prime}}{2}+h} E(v, t) d t-\frac{\delta}{2} .
$$

This contradicts Claim 4. In fact, in the above in order to guarantee that the quantity in (4.8) converge to those in (4.9), we do not need this strong condition on uniform Lipschitz continuity; see the last part of the proof of Claim 1. Thus our Claim 5 follows.

Claim 6. If we choose $V_{i}$ small enough, then $\exists h>0, \kappa_{0}$ large enough, such that for any solution $u_{\kappa}$ of (1.1) with $\kappa>\kappa_{0}$, if $u_{\kappa}\left(\left[T_{1}-h, T_{1}\right]\right)$ and $u_{\kappa}\left(\left[T_{2}, T_{2}+h\right]\right)$ lie in $V_{i}$ (here $T_{2}>T_{1}$ ), then

$$
\int_{T_{2}}^{T_{2}+h} E\left(u_{\kappa}, t\right) d t \leq \int_{T_{1}-h}^{T_{1}} E\left(u_{\kappa}, t\right) d t+\frac{\delta}{4} .
$$

By (4.6), the gradient terms in the above integral can be transformed into those terms containing only $u_{i, \kappa}$. However, $u_{i, \kappa}$ are uniformly Hölder continuous with respect to the parabolic distance, so if we choose $V_{i}$ and $h$ small enough (depending on $\delta$ only), then for any $t \in[0, h]$,

$$
\sup _{\Omega}\left|u_{\kappa}\left(T_{1}-h+t\right)-u_{\kappa}\left(T_{2}+t\right)\right| \leq \frac{\delta}{16} .
$$

At last we note that the last term in (4.6) converges to 0 as $\kappa \rightarrow+\infty$ (using compactness, we can prove that this convergence is uniform, that is, $\forall \epsilon>0, \exists \kappa_{0}$, such that $\forall \kappa>\kappa_{0}$, this term is smaller than $\epsilon>0$ ) and Claim 6 follows.

Now we have the following picture. If $u_{\kappa}\left(t_{0}\right)$ is not in $\bigcup_{i} V_{i}$ because it is close to some connecting orbit $v(t)$, from Claim 1 , we know that after a time $\leq 2 T, u_{\kappa}$ will enter some $V_{i}$. Moreover, its energy decays by an amount at least $-\frac{\delta}{2}$.

If $u_{\kappa}\left(t_{0}\right) \in V_{i}$, then for any $t>t_{0}$, either we have $u_{\kappa}(t) \in V_{i}$ (staying inside and not getting out), and then in view of our arbitrary choice of $V_{i}$, we conclude; or there exists a $t_{1}>t_{0}$ such that $u_{\kappa}\left(t_{1}\right)$ gets outside of $V_{i}$. Then after a time $T$, it will get into some $V_{i}$ again and its energy decays by an amount at least $-\frac{\delta}{2}$. However, we know the energy $E\left(u_{\kappa}, t\right)$ is bounded, so these procedures can happen at most finitely many times, and after some time it will stay in some $V_{i}$ and never get outside of it. That is, there exists a $t^{*}>0$ such that for any $t>t^{*}$, we have $u_{\kappa}(t) \in V_{i}$ for some $i$. In view of the arbitrary choice of $V_{i}$, we conclude. 
The above theorem can be used to prove that:

Corollary 4.3. For $\kappa$ large, any periodic solution of (1.1) must stay near some stationary point of the singular limit system (1.2) in $C^{\alpha}[0,1]$ for any $\alpha \in(0,1)$.

Proof. We just need to note that for a periodic solution, if for large time $t$ it stays in an open neighborhood $V_{i}$ of some $w_{i}$ (the same notation as in the previous theorem), then for all time $t$ it stays in this open neighborhood.

Now we need to study the structure of orbits for $\kappa$ large, near a nontrivial stationary point of the limit equation.

Theorem 4.4. Assume that $w$ is a nontrivial stationary solution of (2.1). Then there exists $\epsilon, \kappa_{0}>0$ such that if $u_{i, \kappa}$ is a solution of (1.1) for $\kappa>\kappa_{0}$ with $\left\|u_{i, \kappa}-w_{i}\right\|_{\infty} \leq \epsilon$ for all large $t$, then $u_{i, \kappa}(t) \rightarrow v_{i}$ uniformly on $[0,1]$ as $t \rightarrow+\infty$, where $v_{i}$ is a nonnegative nontrivial stationary solution of (1.1) with $v_{i}$ near $w_{i}$ (in $L^{\infty}([0,1])$.

Proof. By using omega limit sets, we see that it suffices to prove that the only solutions $u_{\kappa}$ of (1.1) defined for all $t$ and satisfying $\left\|u_{i, \kappa}-w_{i}\right\|_{\infty} \leq \epsilon$ for all $t$ are the constant solutions (stationary in time). Suppose this is false.

We first consider fixed $\kappa$. If $u_{i, \kappa}$ is a nonstationary solution of (1.1) which is bounded for all $t$, standard local parabolic estimates imply that $\frac{\partial u_{i, \kappa}}{\partial t}$ are uniformly bounded (and at least one is nontrivial).

By differentiating the equation in time $t$ we get on $[0,1] \times \mathbb{R}$

$$
\left(\frac{\partial}{\partial t}-\frac{\partial^{2}}{\partial x^{2}}\right) \frac{\partial u_{i, \kappa}}{\partial t}=\left(a_{i}-2 u_{i, \kappa}\right) \frac{\partial u_{i, \kappa}}{\partial t}-\kappa \frac{\partial u_{i, \kappa}}{\partial t} \sum_{j \neq i} u_{j, \kappa}-\kappa u_{i, \kappa} \sum_{j \neq i} \frac{\partial u_{j, \kappa}}{\partial t}
$$

We can rescale $\frac{\partial u_{i, \kappa}}{\partial t}$ to $v_{i, \kappa}$ so that (after a translation in time)

$$
\begin{aligned}
& \sup _{t} \sum_{i} \int_{t}^{t+1} \int_{0}^{1} v_{i, \kappa}^{10}=1, \\
& \sum_{i} \int_{0}^{1} \int_{0}^{1} v_{i, \kappa}^{10} \geq \frac{1}{2} .
\end{aligned}
$$

With this integral bound, by the proof of Proposition 5.15 (in the interior) and Proposition 5.18 (near the boundary), we get

$$
\sup _{[0,1] \times(-\infty,+\infty)} \sum_{i} v_{i, \kappa}^{2} \leq C,
$$

for some constant $C$ independent of $\kappa$.

Now let $\kappa \rightarrow+\infty$. We can prove in some weak sense specified below that

$$
u_{i, \kappa}(x, t) \rightarrow u_{i}(x, t), v_{i, \kappa}(x, t) \rightarrow v_{i}(x, t),
$$

where

(1) $u_{i}(x, t)=w_{i}(x)$. This is because first we have for $\forall t \in(-\infty,+\infty)$

$$
\left\|u_{i}(t)-w_{i}\right\|_{\infty} \leq \epsilon
$$

and then by Proposition 3.12 and the fact that $w$ is isolated we have

$$
\lim _{t \rightarrow \pm \infty} u_{i}(x, t)=w_{i}(x) .
$$


Now we can use the energy identity (Proposition 3.10) to conclude that $\frac{\partial u_{i}}{\partial t} \equiv 0$.

(2) $v_{i}(x, t) \equiv 0$ outside $\left\{w_{i}>0\right\}$. This is an easy consequence of Lemma 5.7, Proposition 5.15 (the case near the free boundary) and Proposition 5.18 (dealing with the boundary point).

(3) $\operatorname{In}\left\{w_{i}>0\right\} \times(-\infty,+\infty)$

$$
\left(\frac{\partial}{\partial t}-\frac{\partial^{2}}{\partial x^{2}}\right) v_{i}=a_{i} v_{i}-2 w_{i} v_{i}
$$

This is because in the equation of $v_{i, \kappa}$, for $j \neq i, u_{j, \kappa}$ and $v_{j, \kappa}$ converge to 0 rapidly in any compact subset of this domain. Thus we can take the limit.

(4) Near the regular part of $\partial\left\{w_{i}>0\right\} \cap \partial\left\{w_{j}>0\right\}$ (here all of the free boundaries are regular: they are lines), we have

$$
\left(\frac{\partial}{\partial t}-\frac{\partial^{2}}{\partial x^{2}}\right)\left(v_{i}-v_{j}\right)=a_{i} v_{i}-2 w_{i} v_{i}-a_{j} v_{j}+2 w_{j} v_{j} .
$$

This can be proved by Corollary 5.14 .

$$
\sum_{i} \int_{0}^{1} \int_{0}^{1} v_{i}^{10} \geq \frac{1}{4}
$$

This can be proved by the combination of (4.12) and (4.13), which implies that for a small $\delta>0$,

$$
\sum_{i} \iint_{[0,1] \times[0,1] \backslash\left\{\sum_{i} w_{i} \leq \delta\right\}} v_{i, \kappa}^{10} \geq \frac{1}{4}
$$

Then because in $[0,1] \times[0,1] \backslash\left\{\sum_{i} w_{i} \leq \delta\right\}, v_{i, \kappa}$ converge to $v_{i}$ uniformly, we can take the limit to get

$$
\sum_{i} \iint_{[0,1] \times[0,1] \backslash\left\{\sum_{i} w_{i} \leq \delta\right\}} v_{i}^{10} \geq \frac{1}{4} .
$$

In our situation, we can rearrange $v_{i}$ so that if we define

$$
v:=v_{1}-v_{2}+\cdots,
$$

then $v$ satisfies

$$
\left(\frac{\partial}{\partial t}-\frac{\partial^{2}}{\partial x^{2}}\right) v=g(x, w) v
$$

with $g(x, w):=(-1)^{i+1}\left(a_{i}-2 w_{i}\right)$, for $x \in\left\{x \in[0,1] \mid w_{i}(x)>0\right\}$. Using the expansion with eigenfunctions of the operator $\frac{d^{2}}{d x^{2}}+g(x, w)$, from the nondegeneracy of (4.1) and the boundedness of $v$, we can show

$$
v(x, t) \equiv 0,
$$

which contradicts (4.16).

Remark 4.5. In the proof of the uniform boundedness, (4.13), from the bound on the $L^{10}$ norm, (4.12), we can also use the Kato inequality, as in [18. That is, by the equations satisfied by $v_{i, \kappa}$, we get

$$
\left(\frac{\partial}{\partial t}-\frac{\partial^{2}}{\partial x^{2}}\right)\left|v_{i, \kappa}\right| \leq\left(a_{i}-2 u_{i, \kappa}\right)\left|v_{i, \kappa}\right|-\kappa\left|v_{i, \kappa}\right| \sum_{j \neq i} u_{j, \kappa}+\kappa u_{i, \kappa} \sum_{j \neq i}\left|v_{j, \kappa}\right| .
$$


Summing over $i$, the last two terms are canceled, and we get

$$
\left(\frac{\partial}{\partial t}-\frac{\partial^{2}}{\partial x^{2}}\right) \sum_{i}\left|v_{i, \kappa}\right| \leq \sum_{i}\left(a_{i}-2 u_{i, \kappa}\right)\left|v_{i, \kappa}\right| .
$$

Then standard parabolic estimates give our bound on $\sup \left|v_{i, \kappa}\right|$.

Unfortunately, it seems impossible to extend this method to the setting

$$
\left(\frac{\partial}{\partial t}-\frac{\partial^{2}}{\partial x^{2}}\right) v_{i, \kappa}=\left(a_{i}-2 u_{i, \kappa}\right) v_{i, \kappa}-\kappa v_{i, \kappa} \sum_{j \neq i} b_{i j} u_{j, \kappa}+\kappa u_{i, \kappa} \sum_{j \neq i} b_{i j} v_{j, \kappa},
$$

where $b_{i j}$ need not equal $b_{j i}$. On the other hand, our proof can be carried out in this case.

Corollary 4.6. Any nontrivial periodic solution of (1.1) for $\kappa$ large must stay near 0. In particular, its sup-norm is small.

Proof. First, the method of Theorem 4.2 gives that for any solution $u_{\kappa}$ of (1.1), which is defined on $(-\infty,+\infty)$, there exists a $T>0$ and a stationary point $w$ of (1.2) such that for any $t>T, u_{\kappa}(t)$ lies near $w$. In our situation of a periodic solution, it implies that for all $t \in(-\infty,+\infty), u_{\kappa}(t)$ lies near $w$. Now Theorem 4.4 says that if $w \neq 0, u_{\kappa}$ must be stationary.

At last we consider the case for those solutions staying near 0 .

Theorem 4.7. With the assumptions (1), (2), and (3), there exist $\epsilon, \kappa_{0}>0$ such that if $u_{i, \kappa}$ is a solution of (1.1) for $\kappa>\kappa_{0}$ with $\left\|u_{i, \kappa}\right\|_{\infty} \leq \epsilon$ for all large $t$, then $u_{i, \kappa}(t)$ converges uniformly on $[0,1]$ as $t \rightarrow+\infty$.

Proof. As in Theorem 4.4, we only need to consider solutions defined on all time. We can assume (by translation in time)

$$
\begin{aligned}
& \sup _{t} \sum_{i} \int_{t}^{t+1} \int_{0}^{1} u_{i, \kappa}^{2}=\epsilon(\kappa), \\
& \sum_{i} \int_{0}^{1} \int_{0}^{1} u_{i, \kappa}^{2} \geq \frac{1}{2} \epsilon(\kappa) .
\end{aligned}
$$

We define $v_{i, \kappa}=\frac{1}{\epsilon(\kappa)} u_{i, \kappa}$, which satisfies

$$
\frac{\partial v_{i, \kappa}}{\partial t}-\frac{\partial^{2} v_{i, \kappa}}{\partial x^{2}}=a_{i} v_{i, \kappa}-\epsilon(\kappa) v_{i, \kappa}^{2}-\kappa \epsilon(\kappa) v_{i, \kappa} \sum_{j \neq i} v_{j, \kappa} \text { on }[0,1] \times \mathbb{R} .
$$

Taking the limit we get three cases:

(1) $\kappa \epsilon(\kappa) \rightarrow 0$;

(2) $\kappa \epsilon(\kappa) \rightarrow+\infty$;

(3) $\kappa \epsilon(\kappa) \rightarrow \lambda$ for some positive constant $\lambda$.

We can prove in all of these cases that $v_{i, \kappa}$ are uniformly $C^{\alpha}$ continuous with respect to the parabolic distances. (The cases (1) and (3) are easy, and we in fact have higher uniform regularity. Case (2) can be proved using the same method of [2] or simply by the blow up method in [10.) So we can say as $\kappa \rightarrow+\infty$ that $v_{i, \kappa} \rightarrow v_{i}$ 
locally uniformly, and they satisfy the limit equation weakly. Moreover, by taking the limit in (4.22) we have

$$
\begin{aligned}
& \sup _{t} \sum_{i} \int_{t}^{t+1} \int_{0}^{1} v_{i}^{2}=1, \\
& \sum_{i} \int_{0}^{1} \int_{0}^{1} v_{i}^{2} \geq \frac{1}{2} .
\end{aligned}
$$

This implies global Lipschitz continuity of $v_{i}$ with respect to the parabolic distance.

We study these cases separately:

Case 1. Here we have the limit equation

$$
\frac{\partial v_{i}}{\partial t}-\frac{\partial^{2} v_{i}}{\partial x^{2}}=a_{i} v_{i} \text { on }[0,1] \times \mathbb{R} .
$$

We can easily prove, using the energy identity, that as $t \rightarrow \pm \infty, v_{i}(x, t)$ converge to the nontrivial stationary solutions. Then by the uniqueness of the solution of (4.5) (because they are linear ODEs) and the energy identity again, we know that $v_{i}(x, t)=v(x)$. This is a nontrivial solution of (4.5), and we get a contradiction to assumption (3).

Case 2. Here the limit equation is

$$
\left\{\begin{array}{l}
\frac{\partial v_{i}}{\partial t}-\frac{\partial^{2} v_{i}}{\partial x^{2}} \leq a_{i} v_{i} \text { on }[0,1] \times \mathbb{R} \\
\left(\frac{\partial}{\partial t}-\frac{\partial^{2}}{\partial x^{2}}\right)\left(v_{i}-\sum_{j \neq i} v_{j}\right) \geq a_{i} v_{i}-\sum_{j \neq i} a_{j} v_{j} \text { on }[0,1] \times \mathbb{R}
\end{array}\right.
$$

where $v_{i}$ have disjoint support.

Here we can prove an energy identity exactly as in Proposition 3.10, and then the same method in Case 1 gives a contradiction to our assumption (1).

Case 3. Here we have the limit equation

$$
\frac{\partial v_{i}}{\partial t}-\frac{\partial^{2} v_{i}}{\partial x^{2}}=a_{i} v_{i}-\lambda v_{i} \sum_{j \neq i} v_{j} \text { on }[0,1] \times \mathbb{R}
$$

for some $\lambda>0$. By defining $\hat{v}_{i}=\lambda v_{i}$, we get

$$
\frac{\partial \hat{v}_{i}}{\partial t}-\frac{\partial^{2} \hat{v}_{i}}{\partial x^{2}}=a_{i} \hat{v}_{i}-\hat{v}_{i} \sum_{j \neq i} \hat{v}_{i} \text { on }[0,1] \times \mathbb{R} .
$$

So this case can be treated similar to 18 (cf. the last part of Theorem 4 on page 484 ) to get a contradiction to assumption (2).

This completes the proof.

Remark 4.8. We will pursue the higher dimensional case in a forthcoming paper. The difficulty in higher dimension is, generally, we do not know if the solutions of the elliptic singular system are finite. We also do not know if there are finitely many critical values of the corresponding functional. 


\section{Approximate Clean Up Lemma}

In this section, we establish some technical results used in the previous section, mainly an "Approximate Clean Up Lemma" (valid in arbitrary dimension; we can continue to consider the problems in higher dimensions in the next papers) and some of its consequences. For the original Clean Up Lemma, see [2]. Since these results are only intended for the application to the main result in Section 4, and the proof is rather technical, at the first reading the reader need only know the conclusions and go directly to Section 4 , and perhaps finally come back to read the details of the proof.

This lemma, in some sense, describes the little invading property of a strongly competing system. We will simply consider the equation as

$$
\frac{\partial u_{i}}{\partial t}-\Delta u_{i}=-\kappa u_{i} \sum_{j \neq i} u_{j} .
$$

(1.1) can be treated with small changes, which we will indicate in the first subsection, because it can be seen as a small perturbation of (5.1).

The proof of the Approximate Clean Up Lemma follows the iteration scheme used in 2]. After establishing this lemma, we also give a linearization version of this lemma (Corollary 5.14 and Proposition 5.15) by establishing a lower bound for the sum of the two dominating species near the regular part of the free boundaries (Proposition 5.13). Finally we also include a boundary version of the Approximate Clean Up Lemma.

In this section, we often say a quantity $\varepsilon(\kappa)$ (depending on $\kappa$ ) converges to 0 rapidly. This means it has the form

$$
e^{-\kappa^{\alpha}}
$$

for some $\alpha>0$.

5.1. Systems with zeroth order terms. In the paper [2], a Clean Up Lemma was established (see their Section 3, Theorem 11) for the following system on a domain in $\mathbb{R}^{n}$ :

$$
\left\{\begin{array}{l}
\Delta u_{i} \geq 0, \\
\Delta\left(u_{i}-\sum_{j \neq i} u_{j}\right) \leq 0, \\
u_{i} \geq 0, \\
u_{i} u_{j}=0 \quad \text { for } i \neq j .
\end{array}\right.
$$

We need to consider a system with zeroth order perturbation. Assume we are in the unit ball $B_{1}(0) \subset \mathbb{R}^{n}$. Then:

$$
\begin{cases}-\Delta u_{i} \leq f_{i}\left(u_{i}\right), & \\ -\Delta\left(u_{i}-\sum_{j \neq i} u_{j}\right) \geq f_{i}\left(u_{i}\right)-\sum_{j \neq i} f_{j}\left(u_{j}\right), & \\ u_{i} \geq 0, & \text { for } i \neq j . \\ u_{i} u_{j}=0 & \end{cases}
$$

Here $f_{i}, 1 \leq i \leq M$, are given a Lipschitz continuous function defined on $\mathbb{R}^{+}$with $f_{i}(u)=a_{i} u-u^{2}$ (we can allow more general nonlinearity, which we do not pursue here). Then we have the following "Clean Up Lemma". 
Theorem 5.1. Assume at $0 \in B_{1}(0)$ that there exists a sequence $\lambda_{k} \rightarrow 0$ such that the vector function $\frac{1}{\lambda_{k}} u\left(\lambda_{k} x\right)$ converges to

$$
\hat{u}_{1}=\alpha x_{1}^{+}, \hat{u}_{2}=\alpha x_{1}^{-}, \hat{u}_{j}=0 \text { for } j>2 .
$$

Then in a neighborhood of 0

$$
\sum_{j>2} u_{j} \equiv 0 .
$$

Remark 5.2. It is easy to see that the above blow up limit satisfies the system (5.2) on $\mathbb{R}^{n}$ (using the fact $f_{i}(0)=0$ ). The proof of this theorem is almost the same as the one in [2], because, after restricting to a small ball and rescaling, (5.3) can be seen as a small perturbation of (5.2).

Now we give the proof of this theorem. We only show the necessary modifications.

First by rescaling $\hat{u}(x)=\frac{1}{R} u(R x)$, we get

$$
-\triangle \hat{u}_{i}(x)=-R \cdot \triangle u_{i}(R x) \leq R f_{i}\left(R \hat{u}_{i}(x)\right) .
$$

Thus, by letting $R$ sufficiently small, we can assume $u$ is defined on $B_{1}$ with the Lipschitz constant of all $f_{i}$ smaller than a small $\theta>0$ (which will be determined later).

By the assumption of Theorem 5.1, we can also assume that in $B_{1}(0)$

$$
\left|u_{1}-x_{1}^{+}\right| \leq h_{0},\left|u_{2}-x_{1}^{-}\right| \leq h_{0}, \sum_{j>2} u_{j} \leq h_{0},
$$

with $h_{0}>0$ small. Then the iteration is defined as (with $R_{0}=1$ )

$$
\left\{\begin{array}{l}
h_{l+1}=h_{l}^{2}, \\
R_{l}=R_{l-1}-h_{l}^{\frac{1}{2}} .
\end{array}\right.
$$

In each ball $B_{R_{l}}$ we decompose $u_{1}-u_{2}=v_{l}+w_{l}$ such that $v_{l}$ satisfies

$$
\begin{cases}-\triangle v_{l}=f_{1}\left(v_{l}^{+}\right)-f_{2}\left(v_{l}^{-}\right), & \text {in } B_{R_{l}}, \\ v_{l}=u_{1}-u_{2}, & \text { on } \partial B_{R_{l}} .\end{cases}
$$

This decomposition is possible because the solution of this equation is unique. In fact, if there exist two solutions $v$ and $\hat{v}$, then

$$
\begin{cases}-\triangle(v-\hat{v})=c(x)(v-\hat{v}), & \text { in } B_{R_{l}}, \\ v-\hat{v}=0, & \text { on } \partial B_{R_{l}},\end{cases}
$$

where for $f(v)=f_{1}\left(v^{+}\right)-f_{2}\left(v^{-}\right)$, if $v(x) \neq \hat{v}(x)$, then

$$
c(x):=\frac{f(v(x))-f(\hat{v}(x))}{v(x)-\hat{v}(x)},
$$

and if $v(x)=\hat{v}(x)$, we simply take $c(x)=0$.

Note that $\|c\|_{L^{\infty}} \leq \theta$ (the Lipschitz constant of $f_{i}$ ). Then $\exists \lambda>0, \forall \varphi \in H_{0}^{1}\left(B_{1}\right)$, we have

$$
\int_{B_{1}}|\nabla \varphi|^{2}-c(x) \varphi^{2} \geq \lambda \int_{B_{1}} \varphi^{2} .
$$

From this we easily see that $v \equiv \hat{v}$, that is, the solution of (5.4) is unique. 
This inequality also implies, maybe with a different $\lambda, \forall \varphi \in H_{0}^{1}\left(B_{1}\right)$,

$$
\int_{B_{1}}|\nabla \varphi|^{2}-c(x) \varphi^{2} \geq \lambda \int_{B_{1}}|\nabla \varphi|^{2} .
$$

With these preliminaries, we have the following lemmas. The first is an easy consequence of the weak maximum principle.

Lemma 5.3. In a ball $B$, for $\varphi \in H^{1}(B)$ satisfying

$$
\begin{cases}-\triangle \varphi-c \varphi \geq 0, & \text { in } B, \\ \varphi \geq 0, & \text { on } \partial B\end{cases}
$$

weakly, then

$$
\varphi \geq 0
$$

in $B$.

Proof. Multiply the equation with $\varphi^{-}:=\max \{-\varphi, 0\}$, integrate by parts, and then use the inequality (5.5).

Next we need to construct a comparison function:

Lemma 5.4. In a ball $B$ with radius $R \in\left[\frac{1}{2}, 1\right]$, for $\varphi \in H^{1}(B)$ satisfying

$$
\begin{cases}-\triangle \varphi-c \varphi=h, & \text { in } B, \\ \varphi=h, & \text { on } \partial B,\end{cases}
$$

where $h$ is a positive constant, then in $B$

$$
0 \leq \varphi \leq C(n, \theta) h
$$

for a constant $C(n, \theta)$ depending only on $n$ and $\theta$.

Proof. That $\varphi \geq 0$ is a consequence of the previous lemma.

By considering $\frac{\varphi}{h}$, we can assume $h=1$. Then with the help of (5.6), we can use the classical De Giorgi iteration to prove the second claim.

At last, we need a lemma concerning the comparison of the gradients of two solutions (or approximate solutions, which can be treated similarly).

Lemma 5.5. If $v_{1}, v_{2}$ are two solutions of (5.4) in $B_{1}$, with

$$
\sup _{B_{1}}\left|v_{1}-v_{2}\right| \leq h
$$

then

$$
\sup _{B_{1-h^{\frac{1}{2}}}}\left|\nabla v_{1}-\nabla v_{2}\right| \leq C h^{\frac{1}{2}} .
$$

Proof. First, differentiating the equation for $v_{i}, i=1,2$, we get

$$
-\triangle \nabla v_{i}=f^{\prime}\left(v_{i}\right) \nabla v_{i} .
$$

Noting here the special form of $f\left(v_{i}\right)$, we can subtract these two equations to get

$$
\begin{aligned}
-\triangle\left(\nabla v_{1}-\nabla v_{2}\right) & =f^{\prime}\left(v_{1}\right)\left(\nabla v_{1}-\nabla v_{2}\right)+\left[f^{\prime}\left(v_{1}\right)-f^{\prime}\left(v_{2}\right)\right] \nabla v_{2} \\
& =f^{\prime}\left(v_{1}\right)\left(\nabla v_{1}-\nabla v_{2}\right)+d(x)\left(v_{1}-v_{2}\right) \nabla v_{2},
\end{aligned}
$$

where

$$
d(x)=-2 v_{1}^{+}+2 v_{1}^{-}+2 v_{2}^{+}-2 v_{2}^{-} .
$$


Next, take a cut-off function $\eta \equiv 1$ in $B_{1-h^{\frac{1}{2}}}, \eta \equiv 0$ outside $B_{1}$. We have

$$
\int\left|\nabla v_{1}-\nabla v_{2}\right|^{2} \eta^{2}+\triangle\left(v_{1}-v_{2}\right)\left(v_{1}-v_{2}\right) \eta^{2}+2 \eta\left(v_{1}-v_{2}\right)\left(\nabla v_{1}-\nabla v_{2}\right) \nabla \eta=0
$$

By our assumptions on $\sup _{B_{1}}\left|v_{1}-v_{2}\right|$, this implies

$$
\int\left|\nabla v_{1}-\nabla v_{2}\right|^{2} \eta^{2} \leq \int\left(C+|\nabla \eta|^{2}\right)\left|v_{1}-v_{2}\right|^{2} \eta^{2} \leq C h .
$$

Combining this with (5.10), we can prove our result by standard Moser iteration using (5.6).

Noting the fact that if we choose $R$ small enough at the beginning (depending on $h_{0}$ ), then

$$
\left|\triangle x_{1}+f_{1}\left(x_{1}^{+}\right)-f_{2}\left(x_{1}^{-}\right)\right| \leq h_{0}, \text { in } B_{1}
$$

by (5.5), and then

$$
\begin{gathered}
\sup _{B_{1}}\left|v_{0}-x_{1}\right| \leq C h_{0}, \\
\sup _{B}\left|\nabla v_{0}-\nabla x_{1}\right| \leq C h_{0}^{\frac{1}{2}},
\end{gathered}
$$

where $C$ depends on $n$ and $\theta$ only. The first estimate can be proved by Moser iteration using (5.6) (cf. page 191 in [19]), and the second estimate can be proved using compactness, analogous to Caffarelli's treatment on perturbation ([1]), provided at the beginning we have chosen the $R$ small enough. (Assume there exists $h_{0}>0$ such that there exists a sequence of $R_{m} \rightarrow 0$ and a sequence of $v_{m, 0}$ constructed as above, but the above estimate is not valid. Then we can pass to the limit to get a contradiction.) Now we can give the following decay estimate.

Lemma 5.6. In $B_{R_{l}}$ we have

(1) $\left|v_{l}-\left(u_{1}-u_{2}\right)\right| \leq h_{l}$.

(2) $\left|\nabla\left(v_{l}-v_{l-1}\right)\right| \leq h_{l-1}^{\frac{1}{2}}$.

(3) $\left|\nabla v_{l}-e_{1}\right| \leq \sum_{j=1}^{l} h_{j-1}^{\frac{1}{2}} \leq \frac{1}{4}$.

(4) The level surface of $v_{l}=0$ is Lipschitz with Lipschitz constant less than 3.

The proof of the decay of $\sum_{j>2} u_{j}$ from $B_{R_{l}}$ to $B_{R_{l+1}}$ is almost the same as in 2], with the only difference being that we now only have

$$
\triangle u_{i} \geq-\theta u_{i}
$$

But from this we can still get a weaker mean value inequality similar to the one for subharmonic functions. 
We also need to show that in $B_{R_{l+1}}, w_{l+1}$ can be controlled by $\sum_{j>2} u_{j}$. This can be seen by the equations they satisfy,

$$
\begin{aligned}
-\triangle w_{l+1} & =\Delta v_{l+1}-\triangle\left(u_{1}-u_{2}\right) \\
& =-f\left(v_{l+1}\right)+f\left(u_{1}-u_{2}\right)-\sum_{i>2} \mu_{1 i}+\sum_{i>2} \mu_{2 i} \\
& =-c(x) w_{l+1}-\sum_{i>2} \mu_{1 i}+\sum_{i>2} \mu_{2 i},
\end{aligned}
$$

where $f$ and $c$ are defined as before, and for $i \neq j, \mu_{i j}$ are Radon measure parts of $\triangle u_{i}$ supported on $\partial\left\{u_{i}>0\right\} \cap \partial\left\{u_{j}>0\right\}$. For $\sum_{j>2} u_{j}$ we have

$$
-\triangle \sum_{j>2} u_{j}=\sum_{j>2} f_{j}\left(u_{j}\right)-\sum_{j>2}\left(\mu_{1 j}+\mu_{2 j}\right) .
$$

Here the first term of the right-hand side has the order $\theta h_{l+1}$ on $B_{R_{l+1}}$. So we have

$$
\begin{gathered}
-\triangle w_{l+1}-c(x) w_{l+1} \geq-\triangle \sum_{j>2} u_{j}-\theta h_{l+1}, \\
-\triangle w_{l+1}-c(x) w_{l+1} \leq \triangle \sum_{j>2} u_{j}+\theta h_{l+1},
\end{gathered}
$$

or in another form

$$
\begin{gathered}
(-\triangle-c(x))\left(w_{l+1}-\sum_{j>2} u_{j}\right) \geq-\theta h_{l+1}, \\
(-\triangle-c(x))\left(w_{l+1}-\sum_{j>2} u_{j}\right) \leq \theta h_{l+1} .
\end{gathered}
$$

From the local interior estimate for the elliptic operator $-\triangle-c(x)$ we get a control

$$
\sup _{B_{R_{l+1}}}\left|w_{l+1}\right| \leq C h_{l+1}
$$

with a constant $C$ depending only on the initial data.

5.2. Proof of the Approximate Clean Up Lemma. First we need a lemma which is the parabolic analogue of Lemma 4.4 in 10. In the following, we may not distinguish the various constants in the estimates, which are simply denoted by $C$ or even omitted if no confusion occurs.

Lemma 5.7. Let $Q_{2 R}:=B_{2 R}(x) \times\left(t-4 R^{2}, t+4 R^{2}\right)$ and $Q_{R}:=B_{R}(x) \times(t-$ $\left.R^{2}, t+R^{2}\right)$. Let $A, M$ be two positive constants and $u$ be a smooth function in $Q_{2 R}$ such that

$$
\begin{cases}\frac{\partial u}{\partial t}-\Delta u \leq-M u, & \text { in } Q_{2 R} \\ u \geq 0, & \text { in } Q_{2 R} \\ u \leq A, & \text { in } Q_{2 R} .\end{cases}
$$

If $M$ is sufficiently large compared to $R$, then we have

$$
\sup _{Q_{R}} u \leq C_{1} A e^{-C_{2} R M^{\frac{1}{2}}},
$$

where $C_{1}, C_{2}$ are positive constants depending only on the dimension. 
Proof. Given two $r_{1}, r_{2}$, take a cut-off function $\eta$ such that

$$
\left\{\begin{array}{r}
\eta \equiv 1, \text { in } Q_{r_{1}} \\
\eta \equiv 0, \text { outside } Q_{r_{2}} \\
\left|\frac{\partial \eta}{\partial t}\right| \leq 10\left(r_{2}-r_{1}\right)^{-2} \\
|\triangle \eta| \leq 10\left(r_{2}-r_{1}\right)^{-2}
\end{array}\right.
$$

First by standard calculation we have

$$
\frac{\partial u^{2}}{\partial t}-\triangle u^{2} \leq-M u^{2}
$$

Multiplying the equation with $\eta$ and integrating by parts, we get

$$
\begin{aligned}
& \iint_{Q_{r_{1}}} u^{2} \leq M^{-1} \iint\left(\triangle u^{2}-\frac{\partial u^{2}}{\partial t}\right) \eta \\
\leq & M^{-1} \iint u^{2}\left(\triangle \eta+\frac{\partial \eta}{\partial t}\right) \\
\leq & 20 M^{-1}\left(r_{2}-r_{1}\right)^{-2} \iint_{Q_{r_{2}}} u^{2} .
\end{aligned}
$$

Now divide the interval $[R, 2 R]$ into $N+1$ equal parts with

$$
r_{k}=\left(1+\frac{k}{N}\right) R, \text { for } k=0,1, \cdots, N
$$

An iteration in $k$ using the above inequality gives

$$
\iint_{Q_{R}} u^{2} \leq C\left(\frac{N^{2}}{R^{2} M}\right)^{N} \iint_{Q_{2 R}} u^{2} .
$$

If $R \sqrt{M}>100$ (sufficiently large is enough) we can take $N=\left[\frac{1}{2} R \sqrt{M}\right]$ (the integer part) to get, for two positive constants $C_{1}, C_{2}$ depending only on the dimension,

$$
\iint_{Q_{R}} u^{2} \leq C_{1} e^{-C_{2} R M^{\frac{1}{2}}} \iint_{Q_{2 R}} u^{2}
$$

Then by the sup-bound estimate of the sub-caloric function (Theorem 6.17, page 121 in [25), we can complete the proof of this lemma.

Theorem 5.8. Assume a sequence of the solution of (5.1), $u_{i, \kappa_{n}}$, converge to $u_{i}$. If we have a point of the free boundaries $(x, t)$, where in a neighborhood there are exactly two components $u_{1}, u_{2}$ which do not vanish, and $\left|\nabla u_{1}(x, t)\right| \neq 0$, then in a small parabolic cylinder $Q_{R}(x, t):=B_{R}(x) \times\left(t-R^{2}, t+R^{2}\right)$ we have

$$
\sup _{Q_{R}} \sum_{i \neq 1,2} u_{i, \kappa_{n}} \leq C(R) e^{-\kappa^{1 / 12}},
$$

for $\kappa$ large, where $C(R)$ is independent of $\kappa$.

Proof. Assume the free boundary point is $(0,0)$. After a rescaling (this changes $\kappa$ by a fixed scale), we may assume we are in $Q_{1}$, and here we have

$$
\left\{\begin{array}{r}
\left(\frac{\partial}{\partial t}-\triangle\right)\left(u_{1}-u_{2}\right)=0, \\
\left|\nabla\left(u_{1}(x, t)-u_{2}(x, t)\right)-e_{1}\right| \leq \epsilon, \\
\nabla\left(u_{1}(0,0)-u_{2}(0,0)\right)=e_{1} .
\end{array}\right.
$$


Here the unit vector $e_{1}=(1,0,0, \cdots, 0) \in \mathbb{R}^{n}$ and $\epsilon$ is a small positive constant. Given an $h_{0}>0$ small enough, which will be chosen later, we know for $\kappa$ large enough that

$$
\begin{gathered}
\sup _{Q_{1}}\left|\left(u_{1, \kappa}-u_{2, \kappa}\right)-\left(u_{1}-u_{2}\right)\right| \leq h_{0} \\
\sup _{Q_{1}}\left|\sum_{i>2} u_{i, \kappa}\right| \leq h_{0} .
\end{gathered}
$$

Define the iteration (starting with $R_{0}=1$ )

$$
\left\{\begin{array}{l}
h_{l+1}=h_{l}^{2}, \\
R_{l}=R_{l-1}-h_{l-1}^{\frac{1}{2}} .
\end{array}\right.
$$

We know that if $h_{0}$ is small enough, then $h_{l}$ will converge to 0 rapidly, and $\lim _{l \rightarrow+\infty} R_{l} \geq \frac{1}{2}$. We need to estimate the decay of $\sup _{Q_{R_{l}}} \sum_{i \neq 1,2} u_{i, \kappa}$.

In the cylinder $Q_{R_{l}}$, we decompose $u_{1, \kappa}-u_{2, \kappa}:=v_{l}+w_{l}$, where

$$
\begin{cases}\left(\frac{\partial}{\partial t}-\triangle\right) v_{l}=0, & \text { in } Q_{R_{l}}, \\ v_{l}=u_{1, \kappa}-u_{2, \kappa}, & \text { on } \partial_{p} Q_{R_{l}}\end{cases}
$$

Note here that as $l=0$, because $\left|v_{0}-\left(u_{1}-u_{2}\right)\right| \leq h_{0}$ on $\partial_{p} Q_{1}$ and both satisfy the heat equation, we then have

$$
\sup _{Q_{1}}\left|v_{0}-\left(u_{1, \kappa}-u_{2, \kappa}\right)\right| \leq 2 h_{0}
$$

In order to complete the proof, we need the following lemma:

Lemma 5.9. If $h_{l} \geq \kappa^{-\frac{1}{6}}$, then in $Q_{R_{l}}$ we have:

(1) $\left|v_{l}-\left(u_{1, \kappa}-u_{2, \kappa}\right)\right| \leq e^{-h_{l-1}^{-\frac{1}{2}}}+h_{l-1} e^{-h_{l-1}^{\frac{3}{2}} \kappa^{\frac{1}{2}}} \leq h_{l-1}^{2}$.

(2) $\left|\nabla\left(v_{l}-v_{l-1}\right)\right| \leq h_{l-1}^{\frac{1}{2}}$.

(3) $\left|\nabla v_{l}-e_{1}\right| \leq \sum_{j=1}^{l} h_{j-1}^{\frac{1}{2}} \leq \frac{1}{4}$.

(4) The level surface of $v_{l}=0$ is Lipschitz with a Lipschitz constant less than 3.

We divide the cylinder $Q_{R_{l+1}}$ into two parts: the good part where $v_{l} \geq 2 h_{l}$ and the bad part where $v_{l} \leq 2 h_{l}$.

In the first part,

$$
u_{1, \kappa}+u_{2, \kappa} \geq\left|u_{1, \kappa}-u_{2, \kappa}\right| \geq v_{l}-h_{l} \geq h_{l}
$$

By the third result in Lemma 5.9 (combining it with a standard a priori estimate of the heat equation, see page 17 in [25]), $v_{l}$ is Lipschitz with a Lipschitz constant less than 3 , so if $v_{l}(x, t) \geq 2 h_{l}$, there is a cylinder of size $\sim h_{l}$ such that on this 
cylinder, $v_{l}(x, t) \geq \frac{1}{2} h_{l}$. Then we can use Lemma 5.7 to get that

$$
\sum_{i \neq 1,2} u_{i, \kappa}(x, t) \leq h_{l} e^{-h_{l}^{\frac{3}{2}} \sqrt{\kappa}}
$$

In the remaining part where $v_{l} \leq 2 h_{l}$, note that it is a narrow domain in the sense of [2], Lemma 24. We have the following estimate:

Lemma 5.10. For $(x, t) \in\left\{v_{l} \leq 2 h_{l}\right\} \cap Q_{R_{l}}$, we have

$$
\sum_{i \neq 1,2} u_{i, \kappa}(x, t) \leq e^{-\frac{R_{l}-\sqrt{t+|x|^{2}}}{h_{l}}} h_{l}+h_{l} e^{-h_{l}^{\frac{3}{2}} \sqrt{\kappa}} .
$$

Proof. Take an integer $N$ such that $\lambda N h_{l} \sim R_{l}-\sqrt{t+|x|^{2}}$ and define $\hat{Q}_{i}:=$ $Q_{R_{l}-i \lambda h_{l}}, i=0,1, \cdots, N$, where $\lambda$ will be determined later. From $\hat{Q}_{i}:=Q_{i} \cap\left\{v_{l} \leq\right.$ $\left.h_{l}\right\}$ to $\hat{Q}_{i+1}$ we have the following decay estimate, using the sup-norm estimate of sub-caloric function:

$$
\begin{aligned}
\sup _{\hat{Q}_{i+1}} u_{i, \kappa}^{2} & \leq C(n) \frac{1}{\left|Q_{\lambda h_{l}}\right|} \iint_{Q_{\lambda h_{l}}} u_{i, \kappa}^{2} \\
& \leq C(n) \frac{1}{\left|Q_{\lambda h_{l}}\right|}\left[\iint_{Q_{\lambda h_{l}} \cap\left\{v_{l} \geq 2 h_{l}\right\}} u_{i, \kappa}^{2}+\iint_{Q_{\lambda h_{l}} \cap\left\{v_{l} \leq 2 h_{l}\right\}} u_{i, \kappa}^{2}\right] \\
& \leq C(n) h_{l} e^{-h_{l}^{\frac{3}{2}} \sqrt{\kappa}}+\frac{1}{2} \sup _{\hat{Q}_{i}} u_{i, \kappa}^{2},
\end{aligned}
$$

if $\left\{v_{l} \leq 2 h_{l}\right\} \cap Q_{R_{l}}$ is narrow in the sense that, for any $(x, t) \in\left\{v_{l} \leq 2 h_{l}\right\} \cap Q_{R_{l}}$ we have

$$
\left|Q_{\lambda h_{l}}(x, t) \cap\left\{v_{l} \leq 2 h_{l}\right\} \cap Q_{R_{l}}\right| \leq \frac{1}{2 C(n)}\left|Q_{\lambda h_{l}}(x, t)\right| .
$$

We can choose $\lambda$, which depends only on the Lipschitz constant of $v_{l}$, to satisfy this condition.

Finally, an iteration of (4.22) in $j$ gives our result. In fact,

$$
\sup _{\hat{Q}_{N}} u_{i, \kappa}^{2} \leq\left(1+\frac{1}{2}+\cdots+\frac{1}{2^{N}}\right) C(n) h_{l} e^{-h_{l}^{\frac{3}{2}} \sqrt{\kappa}}+\frac{1}{2^{N+1}} \sup _{\hat{Q_{0}}} u_{i, \kappa}^{2} .
$$

Now in $Q_{R_{l+1}}$,

$$
\begin{aligned}
\left(\frac{\partial}{\partial t}-\triangle\right) w_{l+1}= & \left(\frac{\partial}{\partial t}-\triangle\right)\left(u_{1, \kappa}-u_{2, \kappa}\right) \\
\leq & \left(\frac{\partial}{\partial t}-\triangle\right) \sum_{i \neq 1,2} u_{i, \kappa}
\end{aligned}
$$

and

$$
\left(\frac{\partial}{\partial t}-\triangle\right) w_{l+1} \geq-\left(\frac{\partial}{\partial t}-\triangle\right) \sum_{i \neq 1,2} u_{i, \kappa}
$$


Here the second inequality follows from (5.18) and (5.19). We also have $w_{l+1}=0$ on $\partial_{p} Q_{R_{l+1}}$, so in $Q_{R_{l+1}}$

$$
\left|w_{l+1}\right| \leq \sum_{i \neq 1,2} u_{i, \kappa} \leq h_{l} e^{-h_{l}^{-\frac{1}{2}}}+h_{l} e^{-h_{l}^{\frac{3}{2}} \sqrt{\kappa}}
$$

This gives the first part of Lemma 5.9.

Now we also have in $Q_{R_{l+1}}$ that

$$
\begin{aligned}
\left|v_{l}-v_{l+1}\right| & \leq\left|v_{l}-\left(u_{1, \kappa}-u_{2, \kappa}\right)\right|+\left|\left(u_{1, \kappa}-u_{2, \kappa}\right)-v_{l+1}\right| \\
& \leq h_{l},
\end{aligned}
$$

and an interior gradient estimate gives the second part of Lemma 5.9 (after shrink$\operatorname{ing} Q_{R_{l+1}}$ by a factor $\left.h_{l}^{\frac{1}{2}}\right)$. The remaining parts are easy consequences of the second part.

From (5.22), we also get, at the end point of our iteration, where $h_{l_{0}}=\kappa^{-\frac{1}{6}}$, in $Q_{R_{l_{0}}}$ with $R_{l_{0}} \geq \frac{1}{2}$

$$
\sum_{i \neq 1,2} u_{i, \kappa} \leq e^{-h_{l_{0}}^{-\frac{1}{2}}}+h_{l_{0}} e^{-h_{l_{0}}^{\frac{3}{2}} \sqrt{\kappa}} \leq e^{-\kappa^{\frac{1}{12}}} .
$$

This ends the proof of Theorem 5.8 .

Remark 5.11. From Proposition 3.8 we know the condition in this theorem is satisfied except for finitely many times $T_{1}, T_{2}, \cdots$, in particular, in $[0,1] \times\left(T_{1},+\infty\right)$.

In 1 dimension, in the case of an elliptic equation (a system of ODEs), the iteration scheme in the proof is not needed (in fact, it can't be applied). We can prove the conclusion directly. That is, if we are in the position of the starting point of the iteration scheme, instead of defining an iteration we estimate $\sum_{i>2} u_{i, \kappa}$ in the two domains where $\left|u_{1, \kappa}-u_{2, \kappa}\right| \geq h$ or $\left|u_{1, \kappa}-u_{2, \kappa}\right| \leq h$, separately.

In the first domain, we have $\kappa\left(u_{1, \kappa}+u_{2, \kappa}\right) \geq \kappa h$, so

$$
\sum_{i>2} u_{i, \kappa} \leq e^{-\kappa h^{\frac{3}{2}}} \text {. }
$$

In the second domain, which we can assume to be $[-h, h], u_{\kappa}$ satisfies (here $\theta$ is the Lipschitz constant of $f_{i}$ )

$$
-u_{i, \kappa}^{\prime \prime} \leq \theta u_{i, \kappa} .
$$

We claim that, for some constant $C>0$,

$$
\sup _{[-h, h]} u_{i, \kappa} \leq \max \left\{u_{i, \kappa}(-h), u_{i, \kappa}(h)\right\}\left(1+C h^{2}\right) .
$$

This can be seen by rescaling $u_{i, \kappa}$ to $\hat{u}_{i, \kappa}$, which is defined on $[-1,1]$ :

$$
\hat{u}_{i, \kappa}(x)=\frac{1}{\max \left\{u_{i, \kappa}(-h), u_{i, \kappa}(h)\right\}} u_{i, \kappa}(h x),
$$

satisfying

$$
-\hat{u}_{i, \kappa}^{\prime \prime} \leq \theta h^{2} \hat{u}_{i, \kappa}
$$


Corollary 5.12. Under the assumption of Theorem [5.8, in $Q_{\frac{R}{2}}(x, t)$, we have for $u_{\kappa}=u_{1, \kappa}-u_{2, \kappa}$ :

$$
\left(\frac{\partial}{\partial t}-\triangle\right) u_{\kappa}=\epsilon(\kappa)
$$

where $\lim _{\kappa \rightarrow+\infty} \epsilon(\kappa)=0$.

5.3. A linearized version. In this and the following subsection we study the convergence of the solutions to the linearized equations of (5.1), under the same assumption of the Approximate Clean Up Lemma. We need these results in the proof of Theorem 4.4 in order to get a nontrivial solution of the linearized system of the limit system as $\kappa \rightarrow+\infty$. (This then contradicts our nondegeneracy assumption; see Section 4.)

We have shown that in a neighborhood of the regular point of the free boundaries, where in the limit case only $u_{1}$ and $u_{2}$ are not vanishing, then for $j \neq 1,2, u_{j, \kappa} \leq$ $e^{-\kappa^{\alpha}}$ for some $\alpha \in(0,1)$. It's easily seen that here we must have

$$
\kappa\left(u_{1, \kappa}+u_{2, \kappa}\right) \rightarrow+\infty .
$$

In the following propositions we will give an explicit lower bound, which also improves the decay rate in Theorem 5.8 a postiori.

Proposition 5.13. Under the assumption of Theorem [5.8, there exists a constant $C$ such that in a small cylinder,

$$
\kappa^{\frac{1}{3}}\left(u_{1, \kappa}+u_{2, \kappa}\right) \geq C .
$$

Proof. We add a remark here that the elliptic case is similar. We assume in a cylinder $Q_{R}$ that $u_{i, \kappa}$ satisfy the equation:

$$
\frac{\partial u_{i, \kappa}}{\partial t}-\Delta u_{i, \kappa}=-\kappa u_{i, \kappa} \sum_{j \neq i} u_{j, \kappa},
$$

and $u_{j, \kappa} \rightarrow 0$ rapidly for $j \neq 1,2 ; u_{\kappa}=u_{1, \kappa}-u_{2, \kappa}$ satisfies

$$
\frac{\partial u_{\kappa}}{\partial t}-\Delta u_{\kappa}=\varepsilon(\kappa)
$$

where $\varepsilon(\kappa) \rightarrow 0$ rapidly; $u_{1, \kappa} \rightarrow u_{1}$ and $u_{2, \kappa} \rightarrow u_{2}$ uniformly in $Q_{R}$, with $u=u_{1}-u_{2}$ caloric in $Q_{R}$; by the interior gradient estimate of caloric functions,

$$
|\nabla u-e| \leq \varepsilon
$$

for a fixed unit vector $e$ and a small $\varepsilon>0$ (this is derived from the assumption of Theorem 5.8, see the third result in Lemma 5.9).

We want to prove that in the cylinder $Q_{\frac{R}{2}}$

$$
\kappa^{\frac{1}{3}}\left(u_{1, \kappa}+u_{2, \kappa}\right) \geq C .
$$

Assume by contrast, then $\exists X_{\kappa} \in Q_{\frac{R}{2}}$, such that

$$
\kappa^{\frac{1}{3}}\left(u_{1, \kappa}+u_{2, \kappa}\right)\left(X_{\kappa}\right)=\epsilon(\kappa),
$$

where $\lim _{\kappa \rightarrow+\infty} \epsilon(\kappa)=0$.

Without loss of generality, we can assume $X_{\kappa} \rightarrow X_{0}$ for some $X_{0} \in Q_{\frac{R}{2}}$. 
From (5.25), we know $u_{\kappa}$ are uniformly bounded, and close to $u$ if $\kappa$ is large, in $C^{1+\alpha, \frac{\alpha}{2}}\left(Q_{R}\right)$ for any $\alpha \in(0,1)$. So without loss of generality, we can assume:

(1) $\left|\nabla u_{\kappa}-e\right| \leq 2 \varepsilon$ and $\left|\nabla u_{\kappa}\right| \geq 1-2 \varepsilon$;

(2) by the implicit function theorem (applied to each time sheet $Q_{r} \cap\{t\}$ ), $\Sigma_{\kappa}:=\left\{u_{\kappa}=0\right\}$ can be represented by the graph of a function $\varphi_{\kappa}$ in the direction $e$, with $\varphi_{\kappa}$ uniformly bounded in $C^{1+\alpha, \frac{\alpha}{2}}\left(Q_{R}\right)$ for some $\alpha \in(0,1)$;

(3) by the lower bound on $\left|\nabla u_{\kappa}\right|,\left|u_{\kappa}(X)\right| \geq \frac{1}{2} \operatorname{dist}\left(X, \Sigma_{\kappa}\right)$.

Now we have

$$
\begin{aligned}
\epsilon(\kappa) \kappa^{-\frac{1}{3}} & \geq\left(u_{1, \kappa}+u_{2, \kappa}\right)\left(X_{\kappa}\right) \\
& \geq\left|u_{\kappa}\left(X_{\kappa}\right)\right| \\
& \geq \frac{1}{2} \operatorname{dist}\left(X_{\kappa}, \Sigma_{\kappa}\right) \\
& \geq \frac{1}{2} \operatorname{dist}\left(X_{\kappa}, Y_{\kappa}\right),
\end{aligned}
$$

where $Y_{k} \in \Sigma_{\kappa}$ satisfies $\operatorname{dist}\left(X_{\kappa}, Y_{\kappa}\right)=\operatorname{dist}\left(X_{\kappa}, \Sigma_{\kappa}\right)$. From the above inequality we know $\operatorname{dist}\left(X_{\kappa}, Y_{\kappa}\right) \rightarrow 0$. In particular, $Y_{\kappa} \rightarrow X_{0}$, too.

Now define the rescaling (here we define the rescaling $\lambda X=\lambda(x, t):=\left(\lambda x, \lambda^{2} t\right)$ )

$$
\hat{u}_{i, \kappa}(X)=\kappa^{\frac{1}{3}} u_{i, \kappa}\left(Y_{\kappa}+\kappa^{-\frac{1}{3}} X\right),
$$

which satisfies the equations

$$
\frac{\partial \hat{u}_{\kappa}}{\partial t}-\Delta \hat{u}_{i, \kappa}=\hat{u}_{i, \kappa} \sum_{j \neq i} \hat{u}_{j, \kappa}
$$

on $Q_{\kappa \frac{1}{3} R}$.

From the rapid decay of $u_{j, \kappa}$ for $j \neq 1,2$, we have

$$
\sup _{Q} \hat{u}_{j, \kappa} \rightarrow 0 \text {. }
$$

We also have

$$
\hat{X}_{\kappa}=\kappa^{\frac{1}{3}}\left(X_{\kappa}-Y_{\kappa}\right) \rightarrow 0
$$

and for $i=1,2$

$$
\hat{u}_{i, \kappa}\left(\hat{X}_{\kappa}\right) \rightarrow 0
$$

and for $\hat{u}_{\kappa}=\hat{u}_{1, \kappa}-\hat{u}_{2, \kappa}$

$$
\left|\nabla \hat{u}_{\kappa}\right|=\left|\nabla u_{\kappa}\right| \geq \frac{1}{2}
$$

From the uniform Lipschitz continuity of $u_{i, \kappa}$ in [28, we have for some constant $C$ independent of $\kappa$ that

$$
\left|\nabla \hat{u}_{i, \kappa}\right|=\left|\nabla u_{i, \kappa}\right| \leq C .
$$

Here we note that the uniform Lipschitz continuity of $u_{i, \kappa}$ was proven in 28] in the symmetric case, that is, $\forall i, j, b_{i j}=b_{j i}$. If $b_{i j}$ is not symmetric, we can use Theorem 5.2 in [10]. That theorem is proven for the case of two equations. Here by noting 
(5.24) and (5.25), locally our system can be reduced to two equations about $u_{1, \kappa}$ and $u_{2, \kappa}$, with error terms exponentially small, so that theorem can still be applied.

Now it's easily seen that

$$
\begin{aligned}
& \frac{\partial \hat{u}_{1, \kappa}}{\partial t}-\triangle \hat{u}_{1, \kappa}=-\hat{u}_{1, \kappa} \hat{u}_{2, \kappa}+\varepsilon(\kappa), \\
& \frac{\partial \hat{u}_{2, \kappa}}{\partial t}-\triangle \hat{u}_{2, \kappa}=-\hat{u}_{1, \kappa} \hat{u}_{2, \kappa}+\varepsilon(\kappa) .
\end{aligned}
$$

This means

$$
\frac{\partial \hat{u}_{\kappa}}{\partial t}-\triangle \hat{u}_{\kappa}=\varepsilon(\kappa) .
$$

Combining these three equations with (5.28) and noting the uniform Lipschitz continuity, we get

(1) $\hat{u}_{i, \kappa} \rightarrow \hat{u}_{i}$ and $\nabla \hat{u}_{i, \kappa} \rightarrow \nabla \hat{u}_{i}$ uniformly on any compact set of $\mathbb{R}^{n} \times$ $(-\infty,+\infty)$ ( for $\kappa$ large enough), $i=1,2$, with

$$
\frac{\partial \hat{u}_{1}}{\partial t}-\triangle \hat{u}_{1}=\frac{\partial \hat{u}_{2}}{\partial t}-\triangle \hat{u}_{2}=-\hat{u}_{1} \hat{u}_{2}
$$

(2) $\hat{u}_{\kappa} \rightarrow \hat{u}$ locally uniformly with

$$
\frac{\partial \hat{u}}{\partial t}-\triangle \hat{u}=0
$$

From (5.28) we get

$$
\hat{u}_{1}(0)+\hat{u}_{2}(0)=\lim _{\kappa \rightarrow+\infty} \hat{u}_{1, \kappa}\left(\hat{x}_{\kappa}\right)+\hat{u}_{2, \kappa}\left(\hat{x}_{\kappa}\right)=0 .
$$

We also have $\hat{u}_{1}, \hat{u}_{2} \geq 0$, so from the Strong Maximum Principle we get

$$
\hat{u}_{1} \equiv \hat{u}_{2} \equiv 0 .
$$

In particular,

$$
\hat{u} \equiv 0 .
$$

But by taking limit in (5.29) we also have

$$
|\nabla \hat{u}| \geq \frac{1}{2}
$$

which is a contradiction, and our claim is proven.

With the help of this proposition we have the following result:

Corollary 5.14. Under the assumption of Theorem [5.8, and if in $Q_{R}(x, t)$, we have that $f_{i, \kappa}$, which is uniformly bounded independent of $\kappa$, satisfy

$$
\left(\frac{\partial}{\partial t}-\triangle\right) f_{i, \kappa}=-\kappa f_{i, \kappa} \sum_{j \neq i} u_{j, \kappa}-\kappa u_{i, \kappa} \sum_{j \neq i} f_{j, \kappa} .
$$

Then in $Q_{\frac{R}{2}}(x, t)$,

$$
f_{j, \kappa} \rightarrow 0 \text { rapidly, for } j \neq 1,2 \text {. }
$$


Moreover,

where $\lim _{\kappa \rightarrow+\infty} \epsilon(\kappa)=0$ rapidly.

$$
\left(\frac{\partial}{\partial t}-\triangle\right)\left(f_{1, \kappa}-f_{2, \kappa}\right)=\epsilon(\kappa),
$$

Proof. For $j \neq 1,2$, we have

$$
\left(\frac{\partial}{\partial t}-\triangle\right) f_{j, \kappa}=-\kappa f_{j, \kappa}\left(u_{1, \kappa}+u_{2, \kappa}\right)+\epsilon(\kappa) .
$$

Then because there exists $C$ such that $\sup \left|f_{j, \kappa}\right| \leq C$, we have

$$
\left(\frac{\partial}{\partial t}-\triangle\right)\left(f_{j, \kappa}\right)^{2} \leq-\kappa\left(f_{j, \kappa}\right)^{2}\left(u_{1, \kappa}+u_{2, \kappa}\right)+\epsilon(\kappa) .
$$

Because $\kappa\left(u_{1, \kappa}+u_{2, \kappa}\right) \geq \kappa^{\frac{2}{3}}$ in a fixed small cylinder, we can proceed as in the proof of Lemma 5.7 to prove that $\left(f_{j, \kappa}\right)^{2}$ converge to 0 rapidly in a small cylinder. This is a local result, and a procedure by covering gives the global result. With this in hand, we have

$$
\left(\frac{\partial}{\partial t}-\triangle\right)\left(f_{1, \kappa}-f_{2, \kappa}\right)=-\kappa\left(f_{1, \kappa}-f_{2, \kappa}\right) \sum_{j \neq 1,2} u_{j, \kappa}-\kappa\left(u_{1, \kappa}-u_{2, \kappa}\right) \sum_{j \neq 1,2} f_{j, \kappa}
$$

and we conclude.

The assumption in this corollary on the sup-norm is not so satisfactory; instead we give the following:

Proposition 5.15. Under the same assumptions as in the previous corollary, except that we only assume $\int_{Q_{R}} \sum_{i}\left|f_{i}\right|^{q}$ for some $q>2(n+3)$ are uniformly bounded, we still have the same conclusion.

Proof. First, for $f_{i, \kappa}^{2}, i>2$, it satisfies

$$
\begin{aligned}
\left(\frac{\partial}{\partial t}-\triangle\right) f_{i, \kappa}^{2} & \leq 2 f_{i, \kappa}\left(\frac{\partial}{\partial t}-\triangle\right) f_{i, \kappa} \\
& \leq-\kappa f_{i, \kappa}^{2} \sum_{j \neq i} u_{j, \kappa}-\kappa u_{i, \kappa} \sum_{j \neq i} f_{j, \kappa} f_{i, \kappa}
\end{aligned}
$$

where, by Proposition 5.13 .

$$
\begin{aligned}
\kappa \sum_{j \neq i} u_{j, \kappa} & \geq \kappa\left(u_{1, \kappa}+u_{2, \kappa}\right) \\
& \geq \kappa^{\frac{2}{3}} .
\end{aligned}
$$

We can proceed as in the proof of Lemma 5.7, multiplying the equation with $\eta$ and integrating by parts to get the following. (Simply denote $f_{i, \kappa}$ by $f, M:=\kappa^{\frac{2}{3}}$ and $\left.g:=\kappa u_{i, \kappa} \sum_{j \neq i} f_{j, \kappa} f_{i, \kappa} \cdot\right)$

$$
\begin{aligned}
& \iint_{Q_{\frac{r_{1}+r_{2}}{2}}} f^{2} \leq M^{-1} \iint\left(\triangle f^{2}-\frac{\partial f^{2}}{\partial t}\right) \eta+g \eta \\
\leq & M^{-1} \iint f^{2}\left(\triangle \eta+\frac{\partial \eta}{\partial t}\right)+g \eta \\
\leq & M^{-1}\left\{20\left(r_{2}-r_{1}\right)^{-2} \iint_{Q_{r_{2}}} f^{2}+\iint|g|\right\} .
\end{aligned}
$$


Define $R_{k}=\frac{R}{2}\left(2-\frac{k}{N}\right)$ for $k=0,1, \cdots, N$. We use the above estimate to iterate. Then if $M$ is large enough compared to $R$ (exactly as in Lemma 5.7) we get, for $i>2$,

$$
\begin{aligned}
\iint_{Q_{\frac{R}{2}}} f_{i, \kappa}^{2} & \leq e^{-c(n) \kappa^{\frac{1}{3}} R^{2}} \iint_{Q_{R}} f_{i, \kappa}^{2}+C(n) \iint_{Q_{R}} \kappa u_{i, \kappa} \sum_{j \neq i}\left|f_{j, \kappa}\right|\left|f_{i, \kappa}\right| \\
& \leq e^{-\kappa^{\alpha}}
\end{aligned}
$$

for a small $\alpha>0$, because in $Q_{R}, u_{i, \kappa} \leq e^{-\kappa^{\beta}}$ for some $\beta>0$.

By the uniform boundedness of $\iint_{Q_{R}}\left|f_{i, \kappa}\right|^{q}$ and with the help of Hölder inequality, this implies, $\forall 2<p<q, \exists \alpha(p)$ such that

$$
\iint_{Q_{\frac{R}{2}}}\left|f_{i, \kappa}\right|^{p} \leq e^{-\kappa^{\alpha(p)}}
$$

Now in $Q_{\frac{R}{2}}$,

$$
\left(\frac{\partial}{\partial t}-\triangle\right) f_{i, \kappa}^{2} \leq-\kappa u_{i, \kappa} \sum_{j \neq i} f_{j, \kappa} f_{i, \kappa}
$$

Here we can use the standard De Giorgi estimate (see [22]) to get

$$
\begin{aligned}
\sup _{Q_{\frac{R}{4}}} f_{i, \kappa}^{2} & \leq C(n)\left[\left(R^{-n-2} \int_{Q_{\frac{R}{2}}}\left|f_{i, \kappa}\right|^{4}\right)^{\frac{1}{n+3}}+\left(R^{-n-2} \int_{Q_{\frac{R}{2}}}\left|\kappa u_{i, \kappa} \sum_{j \neq i} f_{j, \kappa} f_{i, \kappa}\right|^{n+3}\right)^{\frac{1}{2}}\right] \\
& \leq e^{-\kappa^{\alpha}}
\end{aligned}
$$

for another $\alpha>0$.

Next let's consider $f_{i, \kappa}$ for $i=1,2$, in $Q_{\frac{R}{4}}$. By Kato inequality

$$
\left\{\begin{array}{l}
\left(\frac{\partial}{\partial t}-\triangle\right)\left|f_{1, \kappa}\right| \leq-\kappa\left|f_{1, \kappa}\right| u_{2, \kappa}-\kappa u_{1, \kappa}\left|f_{2, \kappa}\right|+\epsilon(\kappa) \\
\left(\frac{\partial}{\partial t}-\triangle\right)\left|f_{2, \kappa}\right| \leq-\kappa\left|f_{2, \kappa}\right| u_{1, \kappa}-\kappa u_{2, \kappa}\left|f_{1, \kappa}\right|+\epsilon(\kappa)
\end{array}\right.
$$

Summing these two equations we get

$$
\left(\frac{\partial}{\partial t}-\triangle\right)\left(\left|f_{1, \kappa}\right|+\left|f_{2, \kappa}\right|\right) \leq \epsilon(\kappa)
$$

By a standard parabolic estimate, $f_{1, \kappa}$ and $f_{2, \kappa}$ are uniformly bounded in $Q_{\frac{R}{8}}$. The rest of the proof is the same as the previous corollary.

Remark 5.16. If the equation has the form

$$
\left(\frac{\partial}{\partial t}-\triangle\right) f_{i, \kappa}=-\kappa f_{i, \kappa} \sum_{j \neq i} b_{i j} u_{j, \kappa}-\kappa u_{i, \kappa} \sum_{j \neq i} b_{i j} f_{j, \kappa}
$$


the proof is also valid. We only need to make some changes in the place where we used the Kato inequality. We modify $f_{1, \kappa}$ and $f_{2, \kappa}$ into

$$
\left\{\begin{array}{l}
\hat{f}_{1, \kappa}:=b_{21} f_{1, \kappa}, \\
\hat{f}_{2, \kappa}:=b_{12} f_{2, \kappa},
\end{array}\right.
$$

which satisfy

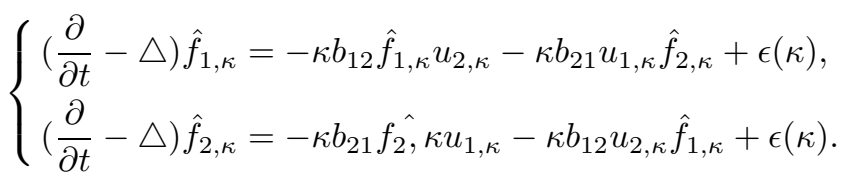

Then we can use the Kato inequality again.

5.4. A boundary version. In this subsection, first we consider

$$
\left(\frac{\partial}{\partial t}-\triangle\right) u_{i}=f_{i}\left(u_{i}\right)-\kappa u_{i} \sum_{j \neq j} u_{j}
$$

near the boundary, with the same assumptions on $f_{i}$ as before. Then we consider its linearization,

$$
\left(\frac{\partial}{\partial t}-\triangle\right) v_{i}=f_{i}^{\prime}\left(u_{i, \kappa}\right) v_{i}-\kappa v_{i} \sum_{j \neq j} u_{j}-\kappa u_{i} \sum_{j \neq i} v_{j},
$$

with $v_{i}=0$ on $\partial \Omega \times \mathbb{R}$.

First, we want to know, for the equation (5.37), that if the solutions $u_{i, \kappa}$ converge to $u_{i}$ as $\kappa \rightarrow+\infty$, such that in $Q_{R} \cap(\Omega \times \mathbb{R}), u_{1}>0$, then what is the behavior of $u_{i, \kappa}$ ? We have the following result:

Proposition 5.17. Under the above assumptions, for $\kappa$ large enough, $\sup _{B_{R} \cap \Omega} \sum_{i>1} u_{i}$ converge to 0 rapidly.

Proof. As in Subsection 5.1, we assume we are in $Q_{1} \cap(\Omega \times \mathbb{R})$, with the Lipschitz constant of $f_{i}$ smaller than a small constant $\theta>0$, and for a small $h_{0}>0$,

$$
\sup _{Q_{1} \cap(\Omega \times \mathbb{R})}\left|u_{1, \kappa}-u_{1}\right| \leq h_{0} .
$$

Define the iteration (starting with $R_{0}=1$ ) as

$$
\left\{\begin{array}{l}
h_{l+1}=h_{l}^{2} \\
R_{l}=R_{l-1}-h_{l}^{\frac{1}{2}}
\end{array}\right.
$$

In each $Q_{R_{l}} \cap(\Omega \times \mathbb{R})$, we can decompose $u_{1, \kappa}:=v_{l}+w_{l}$ such that

$$
\begin{cases}\left(\frac{\partial}{\partial t}-\triangle\right) v_{l}=f_{1}\left(v_{l}\right), & \text { in } Q_{R_{l}} \cap(\Omega \times \mathbb{R}), \\ v_{l}=u_{1, \kappa}, & \text { on } \partial_{p}\left(Q_{R_{l}} \cap(\Omega \times \mathbb{R})\right) .\end{cases}
$$

As in Subsection 5.1, such a $v_{l}$ exists and is unique; moreover, $v_{l}>0$ in $Q_{R_{l}} \cap(\Omega \times \mathbb{R})$.

For $l=0$, from the stability and interior gradient estimate, we have

$$
\begin{cases}\sup _{B_{1} \cap \Omega}\left|v_{0}-u_{1}\right| \leq 2 h_{0}, & \text { in } Q_{1} \cap(\Omega \times \mathbb{R}), \\ \left|\nabla v_{0}-\nabla u_{1}\right| \leq h_{0}^{\frac{1}{2}}, & \text { in } Q_{1-h_{0}^{\frac{1}{2}}} \cap(\Omega \times \mathbb{R}),\end{cases}
$$


which implies, in $Q_{1-h_{0}^{\frac{1}{2}}} \cap(\Omega \times \mathbb{R})$ (here the distance is in the space direction, that is, in a time slice),

$$
v_{0}(x) \geq \operatorname{Cdist}(x, \partial \Omega \times \mathbb{R}) .
$$

Then we can proceed as before, and we can divide $Q_{1-h_{0}^{\frac{1}{2}}} \cap(\Omega \times \mathbb{R})$ into two domains: where $v_{1} \geq C h_{0}$ (then $u_{1, \kappa} \geq C h_{0}$ ), and where $v_{1} \leq C h_{0}$, which is a narrow domain with width $h_{0}$, and we can estimate the decay of $\sum_{j>1} u_{j, \kappa}$ when restricted to $Q_{1-2 h_{0}^{\frac{1}{2}}} \cap(\Omega \times \mathbb{R})$. The details are exactly as before, and we will not repeat them here.

Proposition 5.18. Under the assumptions of the previous proposition, and if in $Q_{R} \cap(\Omega \times \mathbb{R}), v_{i, \kappa}$, are solutions of (5.38), satisfying the fact that

$$
\sum_{i} \int_{Q_{R} \cap(\Omega \times \mathbb{R})}\left|v_{i, \kappa}\right|^{q}
$$

are uniformly bounded for some $q>3 n$, then in $Q_{\frac{R}{4}} \cap(\Omega \times \mathbb{R})$,

$$
v_{j, \kappa} \rightarrow 0 \text { rapidly, for } j \neq 1 \text {. }
$$

Moreover,

$$
\left(\frac{\partial}{\partial t}-\triangle\right) v_{1, \kappa}=f_{1}^{\prime}\left(u_{1, \kappa}\right) v_{1, \kappa}+\epsilon(\kappa),
$$

where $\lim _{\kappa \rightarrow+\infty} \epsilon(\kappa)=0$ rapidly.

Proof. First, for $i>1$,

$$
\begin{aligned}
\left(\frac{\partial}{\partial t}-\triangle\right) v_{i, \kappa}^{2} & \leq f_{i}^{\prime}\left(u_{i, \kappa}\right) v_{i, \kappa}^{2}-\kappa v_{i, \kappa}^{2} \sum_{j \neq j} u_{j}-\kappa u_{i} \sum_{j \neq i} v_{i, \kappa} v_{j, \kappa} \\
& \leq f_{i}^{\prime}\left(u_{i, \kappa}\right) v_{i, \kappa}^{2}-\epsilon(\kappa) \sum_{j \neq j} v_{i, \kappa} v_{j, \kappa} .
\end{aligned}
$$

Standard parabolic estimate (cf. [22]), and noting here the boundary condition for $v_{i, \kappa}$, gives, for $i>1$,

$$
\sup _{Q_{\frac{R}{2}} \cap(\Omega \times \mathbb{R})}\left|v_{i, \kappa}\right| \leq C,
$$

for some constant $C$.

Next, because $u_{1}(x) \geq C \operatorname{dist}(x, \partial \Omega), \forall h>0, \forall x \in\{x: \operatorname{dist}(x, \partial \Omega) \geq 2 C h\}$, there exists a cylinder $Q_{h}(x)$, where $u_{1}(x) \geq h$, so by Lemma 5.7 and the first inequality in (5.41), we get

$$
v_{i, \kappa}^{2}(x) \leq e^{-\kappa h^{3}} .
$$

While for $x \in\{x: \operatorname{dist}(x, \partial \Omega) \leq 2 C h\}$, noting that this is a narrow domain in the sense as before and using the second inequality in (5.41), we have a decay from $Q_{\frac{R}{2}-k h} \cap(\Omega \times \mathbb{R})$ to $Q_{\frac{R}{2}-(k+1) h} \cap(\Omega \times \mathbb{R}), k=0,1,2, \cdots,\left[\frac{R}{4 h}\right]$, so we get

$$
\begin{aligned}
\sup _{Q_{\frac{R}{4}} \cap\{x: 0<\operatorname{dist}(x, \partial \Omega) \leq 2 C h\}} v_{i, \kappa}^{2}(x) \leq 2^{-R h^{-1}} & \sup _{Q_{\frac{R}{2}} \cap\{x: 0<\operatorname{dist}(x, \partial \Omega) \leq 2 C h\}} v_{i, \kappa}^{2} \\
& +\sup _{Q_{\frac{R}{2}} \cap\{x: \operatorname{dist}(x, \partial \Omega) \geq 2 C h\}} v_{i, \kappa}^{2} .
\end{aligned}
$$


If we choose $h=\kappa^{-\frac{1}{4}}$, we then get the first claim in the proposition. Then the second claim is easily seen.

Remark 5.19. In 1 dimension and the elliptic case, the above iteration does not work. We need to use the modification introduced in Remark 5.11. Note that we have boundary value 0 in the above proposition.

\section{ACKNOWLEDGEMENT}

The authors thank the referees very much for their careful reading and very useful suggestions.

\section{REFERENCES}

[1] L.A. Caffarelli, X. Cabre, Fully Nonlinear Elliptic Equations, American Mathematical Society Colloquium Publications 43, American Mathematical Society, Providence, RI, 1995. MR1351007 (96h:35046)

[2] L. A. Caffarelli, A. L. Karakhanyan, F. Lin, The geometry of solutions to a segregation problem for non-divergence systems, J. Fixed Point Theory Appl. 5 (2009), no. 2, 319-351. MR2529504(2010j:35036)

[3] L. A. Caffarelli, F. Lin, An optimal partition problem for eigenvalues, J. Sci. Comput. 31(1)(2007), 5-18. MR2304268 (2008c:65304)

[4] L. A. Caffarelli, F. Lin,, Singularly perturbed elliptic systems and multi-valued harmonic functions with free boundaries, Journal of the American Mathematical Society 21 (2008), 847-862. MR2393430 (2009b:35073)

[5] L. A. Caffarelli, F. Lin, Nonlocal heat flows preserving the $L^{2}$ energy, Discrete and Continuous Dynamical Systems (DCDS-A), 23(1-2) (2009), 49-64. MR2449068 (2009m:35218)

[6] Xu-Yan Chen, A strong unique continuation theorem for parabolic equations, Math. Ann. 311(4) (1998), 603-630. MR1637972 (99h:35078)

[7] M. Conti, S. Terracini, G. Verzini, Nehari's problem and competing species systems, Ann. Inst. H. Poincaré Anal. Non Linéaire 19(6) (2002), 871-888. MR1939088 (2003i:35084)

[8] M. Conti, S. Terracini, G. Verzini, An optimal partition problem related to nonlinear eigenvalues, J. Funct. Anal. 198 (2003), no. 1, 160-196. MR1962357 (2004h:35171)

[9] M. Conti, S. Terracini, G. Verzini, A variational problem for the spatial segregation of reaction diffusion systems, Indiana Univ. Math. J. 54(3) (2005), 779-815. MR2151234(2006b:35180)

[10] M. Conti, S. Terracini, G. Verzini, Asymptotic estimates for the spatial segregation of competitive systems, Adv. Math. 195 (2005), no. 2, 524-560. MR2146353(2006b:35077)

[11] M. Conti, S. Terracini, G. Verzini, Uniqueness and Least Energy Property for Strongly Competing Systems, Interfaces and Free Boundaries 8 (2006), 437-446. MR2283921|(2007j:35045)

[12] E. N. Dancer, Competing species systems with diffusion and large interaction, Rend. Sem. Mat. Fis. Milano 65 (1995), 23-33. MR1459414(99d:92023)

[13] E. N. Dancer, Y.H. Du, Competing species equations with diffusion, large interactions, and jumping nonlinearities, J. Differential Equations 114 (1994), 434-475. MR1303035 (95i:35296)

[14] E. N. Dancer, Yihong Du, Positive solutions for a three-species competition system with diffusion I. General existence results, Nonlinear Analysis 24(3) (1995), 337-357. MR1312772 (96c:92028)

[15] E. N. Dancer, Yihong Du, Positive solutions for a three-species competition system with diffusion II: The case of equal birth rates, Nonlinear Analysis 24(3) (1995), 359-373. MR 1312773 (96c:92029)

[16] E. N. Dancer, Y.H. Du, On a free boundary problem arising from population biology, Indiana Univ. Math. J. 52(1) (2003), 51-67. MR1970020(2004c:35423)

[17] E. N. Dancer, Z.M. Guo, Uniqueness and stability for solutions of competing species equations with large interactions, Comm. Appl. Nonlinear Anal. 1 (1994), 19-45. MR 1280113 (95i:35159)

[18] E. N. Dancer, Zhitao Zhang, Dynamics of Lotka-Volterra competition systems with large interaction, Journal of Differential Equations 182(2) (2002), 470-489. MR1900331 (2003c:35064) 
[19] D. Gilbarg, N. Trudinger, Elliptic partial differential equations of second order, SpringerVerlag Berlin Heidelberg, 2001. MR.1814364 (2001k:35004)

[20] M. Gromov, R. Schoen, Harmonic maps into singular spaces and p-adic superrigidity for lattices in groups of rank one, Publications Mathématiques de L'IHÉS 76(1) (1992), 165-246. MR.1215595 (94e:58032)

[21] Qing Han, F. Lin, Nodal Sets of Solutions of Elliptic Differential Equations, books available on Han's homepage.

[22] Olga A. Ladyzenskaja, V.A. Solonnikov, and Nina N. Ural'ceva, Linear and Quasilinear Equations of Parabolic Type, Volume 23, Translations of Mathematical Monographs, American Mathematical Society, Providence, R.I., 1968. MR.0241822 (39:3159b)

[23] Anthony W. Leung, Systems of nonlinear partial differential equations: Applications to biology and engineering, Kluwer Academic Publishers, Dordrecht, 1989. MR.1621827(99m:35245)

[24] F. Lin, X. Yang, Geometric measure theory - an introduction, Advanced Mathematics (Beijing/Boston), 1. Science Press, Beijing; International Press, Boston, MA, 2002. MR2030862 (2005a:28001)

[25] Gary M. Lieberman, Second order parabolic differential equations, World Scientific, 2005. MR:1465184 (98k:35003)

[26] Chi-Cheung Poon, Unique continuation for parabolic equations, Communications in Partial Differential Equations 21(3-4) (1996), 521-539. MR1387458 (97f:35081)

[27] S. Smale, On the differential equations of species in competition, J. Math. Biology 3 (5-7) (1976). MR0406579 (53:10366)

[28] Kelei Wang, Zhitao Zhang, Some new results in competing systems with many species, Annales de l'Institut Henri Poincaré, Non Linear Analysis 27(2) (2010), 739-761. MR 2595199

School of Mathematics and Statistics, University of Sydney, NSW 2006 Australia

E-mail address: normd@maths.usyd.edu.au

Academy of Mathematics and Systems Science, The Chinese Academy of Sciences, Beijing 100190, People's Republic of China

E-mail address: wangkelei05@mails.gucas.ac.cn

Current address: School of Mathematics and Statistics, University of Sydney, NSW 2006 Australia

E-mail address: kelei@maths.usyd.edu.au

Academy of Mathematics and Systems Science, The Chinese Academy of Sciences, Beijing 100190, People's Republic of China

E-mail address: zzt@math.ac.cn 